\title{
The Potential of Hybrid Pixel Detectors in the Search for the Neutrinoless Double-Beta Decay of ${ }^{116} \mathrm{Cd}$
}

\author{
Thilo Michel, ${ }^{1}$ Thomas Gleixner, ${ }^{1}$ Jürgen Durst, ${ }^{2}$ \\ Mykhaylo Filipenko, ${ }^{1}$ and Stefan Geißelsöder ${ }^{1}$ \\ ${ }^{1}$ Erlangen Centre for Astroparticle Physics (ECAP), Friedrich-Alexander-Universität Erlangen-Nürnberg, Erwin-Rommel-Str. 1, \\ 91058 Erlangen, Germany \\ ${ }^{2}$ Faculty of Physics, Ludwig-Maximilians-Universität München, Schellingstr. 4, 80799 München, Germany
}

Correspondence should be addressed to Thilo Michel; thilo.michel@physik.uni-erlangen.de

Received 28 June 2013; Revised 17 September 2013; Accepted 19 September 2013

Academic Editor: Vincenzo Flaminio

Copyright (C) 2013 Thilo Michel et al. This is an open access article distributed under the Creative Commons Attribution License, which permits unrestricted use, distribution, and reproduction in any medium, provided the original work is properly cited.

\begin{abstract}
We investigated the potential of the energy resolving hybrid pixel detector Timepix contacted to a CdTe sensor layer for the search for the neutrinoless double-beta decay of ${ }^{116} \mathrm{Cd}$. We found that a CdTe sensor layer with $3 \mathrm{~mm}$ thickness and $165 \mu \mathrm{m}$ pixel pitch is optimal with respect to the effective Majorana neutrino mass $\left(m_{\beta \beta}\right)$ sensitivity. In simulations, we were able to demonstrate a possible reduction of the background level caused by single electrons by approximately $75 \%$ at a specific background rate of $10^{-3}$ counts/(kg $\times \mathrm{keV} \times \mathrm{yr})$ at a detection efficiency reduction of about $23 \%$ with track analysis employing random decision forests. Exploitation of the imaging properties with track analysis leads to an improvement in sensitivity to $m_{\beta \beta}$ by about $22 \%$. After 5 years of measuring time, the sensitivity to $m_{\beta \beta}$ of a $420 \mathrm{~kg} \mathrm{CdTe}$ experiment $\left(90 \%{ }^{116} \mathrm{Cd}\right.$ enrichment) would be $59 \mathrm{meV}$ on a $90 \%$ confidence level for a specific single-electron background rate of $10^{-3}$ counts/(kg $\left.\times \mathrm{keV} \times \mathrm{yr}\right)$. The $\alpha$-particle background can be suppressed by at least about six orders of magnitude. The benefit of the hybrid pixel detector technology might be increased significantly if drift-time difference measurements would allow reconstruction of tracks in three dimensions.
\end{abstract}

\section{Introduction}

The question of whether or not neutrinos are their own antiparticles still has not been answered. In many experiments, researchers have to cope with large backgrounds to be able to see if neutrinoless double-beta decay $(0 \nu \beta \beta)$ occurs in nature or not. An observation of one single neutrinoless double-beta decay would directly prove the Majorana character of neutrinos via the Schechter-Valle theorem [1]. Besides this, a measurement of the decay rate $\Gamma$ (or the halflife $T_{1 / 2}^{0 v}=\Gamma^{-1}$ ) of neutrinoless double-beta decay allows the determination of the effective Majorana neutrino mass which is given by

$$
m_{\beta \beta}=\frac{m_{e}}{\sqrt{T_{1 / 2}^{0 v} \times G_{0 v}\left(Q_{\beta \beta}, Z\right) \times\left|M_{0 \nu}\right|^{2}}}=\left|\sum_{i=1}^{3} U_{e i}^{2} \times m_{i}\right|,
$$

where $m_{e}$ is the electron rest mass, $G_{0 \nu}\left(Q_{\beta \beta}, Z\right)$ is the calculable factor for the decay specific phase-space volume,
$\left|M_{0 \gamma}\right|^{2}$ is also the calculable nuclear matrix element, and the $U_{e i}$ are elements of the mixing matrix $\widehat{U}$ which describes neutrino mixing of the three mass eigenstates with masses $m_{i}$ to the electron neutrino. $Z$ is the nuclear charge and $Q_{\beta \beta}$ is the $Q$-value of the double-beta decay. Thus, a measurement of the half-life of $0 \nu \beta \beta$-decay gives information on the masses of the mass eigenstates and the elements of the mixing matrix $\widehat{U}$ which depends on the mixing angles $\theta_{12}, \theta_{23}, \theta_{13}$ and potentially on $\mathrm{CP}$-violating phases [2]. If neutrinos are Majorana fermions, two Majorana phases $\alpha_{1}$ and $\alpha_{2}$ appear in the mixing matrix in addition to the Dirac phase $\delta$. In the Majorana case the mixing matrix reads $\widehat{U}=\widehat{U}_{\text {PMNS }} \times$ diag $\left(1, \alpha_{1}, \alpha_{2}\right)$ where $\widehat{U}_{\text {PMNS }}$ is the Standard Model PMNSmatrix describing Dirac neutrinos and diag $\left(1, \alpha_{1}, \alpha_{2}\right)$ is a $3 \times 3$ diagonal matrix with the entries $1, \alpha_{1}, \alpha_{2}$ on the main diagonal.

On a microscopic scale, the signatures of double-beta decays (neutrino accompanied and neutrinoless) are different 
from most other reactions that might also release energy in the region of the $Q$-value of the double-beta decay: two electrons start simultaneously at one location and travel through the detector materials. In contrast to neutrino accompanied double-beta decay $(2 \nu \beta \beta)$, the summed kinetic energies of the two electrons from $0 \nu \beta \beta$-decay equal the total energy released in the decay ( $Q$-value). Energy resolution, background reduction, and mass increase are the keys for improving sensitivity to the effective Majorana neutrino mass. If a detector could be built, that is, able to "image" the two electrons starting at one location, most background particles would be eliminated: $\alpha$-particles, Compton scattered electrons, electrons and positrons from beta decays $\left(\beta^{-}, \beta^{+}\right)$, and muons. Beta decays to excited states with subsequent internal conversion of $\gamma$-line photons could still have a similar track topology but could be identified because the internal conversion electron has a defined energy.

The experiments SuperNEMO [3] and the Neutrino Xenon TPC (NEXT) $[4,5]$ plan to perform tracking with or in gaseous detectors. Semiconductor detector materialswhich can in principle serve as sensor materials in hybrid pixel detectors-are used in the experiments GERDA [6], Majorana [7], and COBRA [8]. The tracking option with CdTe semiconductor pixel detectors has been evaluated as an additional possible route in the COBRA collaboration [8]. The "Current status and perspectives of the COBRA experiment" are described in detail also in this special issue of Advances in High Energy Physics by Fritts et al. [9]. Background studies with silicon sensor pixel detectors have already been presented in [10]. Hybrid pixel detectors are also an interesting option for the still missing direct detection of neutrino accompanied double electron capture [11].

Hybrid pixel detectors comprise pixelated semiconductor sensor layers for particle detection. We are thus restricted to double-beta decay nuclides of elements which can compose semiconducting materials. The technology of hybrid pixel detectors with silicon sensors is mature. The development of hybrid pixel detectors connected to Germanium sensors is still ongoing. CdTe and $\mathrm{Cd}(\mathrm{Zn}) \mathrm{Te}$ fine pitch hybrid pixel detectors have already been developed, mainly for X-ray imaging for energies from $3 \mathrm{keV}$ to $150 \mathrm{keV}$. The elements cadmium, tellurium, and zinc offer several isotopes that decay via $\beta^{-} \beta^{-}, \beta^{+} \beta^{+}, \beta^{+} \mathrm{EC}$, ECEC: ${ }^{70} \mathrm{Zn},{ }^{114} \mathrm{Cd},{ }^{116} \mathrm{Cd},{ }^{128} \mathrm{Te},{ }^{130} \mathrm{Te},{ }^{106} \mathrm{Cd},{ }^{64} \mathrm{Zn},{ }^{120} \mathrm{Te}$, ${ }^{108} \mathrm{Cd} .{ }^{116} \mathrm{Cd}$ decays via the neutrino accompanied $\beta^{-} \beta^{-}$decay to the ground state of ${ }^{116} \mathrm{Sn}$ with the largest $Q$-value of $2.814 \mathrm{MeV}$ in the $\mathrm{Cd}$, Te, $\mathrm{Zn}$ complex. The phase-space factor for the $0 \nu \beta \beta$-decay of ${ }^{116} \mathrm{Cd}$ is $G_{0 \nu}\left(Q_{\beta \beta}, Z\right)=4.68 \times 10^{-14} \mathrm{yr}^{-1}$ [12]. The matrix element $\left|M_{0 v}\right|$ is about 3.5 [13], but the values given in the literature vary strongly depending on the calculation model. The halflife of the $2 \nu \beta \beta$-decay was measured by Bongrand et al. [14] to be $T_{1 / 2}^{2 v}=[2.88 \pm 0.04$ (stat.) \pm 0.16 (syst.) $] \times 10^{19} \mathrm{yr}$. Danevich et al. [15] have set a limit of $T_{1 / 2}^{0 v}=1.7 \times 10^{23} \mathrm{yr}$ to the half-life of the $0 \nu \beta \beta$-decay channel. The COBRA-experiment (Cadmium Zinc Telluride 0-neutrino double-beta Research Apparatus) proposed by Zuber [8] is located in the Gran Sasso National Laboratory (LNGS). COBRA focuses on the possible detection of the $0 \nu \beta \beta$-decay in ${ }^{116} \mathrm{Cd}$ with semiconducting CdZnTe detectors, whereby the cadmium will be enriched in the isotope ${ }^{116} \mathrm{Cd}$. The original proposal of Zuber [8] is focused on the use of many monolithic, cubic, CdZnTe detectors with medium size of a few $\mathrm{cm}^{3}$. They are larger in volume than the typical sensor layer of a pixel detector but smaller than the germanium diodes employed in the GERDA experiment [6]. Each CdZnTe crystals should be read out individually. This option is the main route of the COBRA collaboration, because experimental complexity and technical challenges are less demanding than in the case of a large-scale experiment with $420 \mathrm{~kg}$ of CdTe pixel detectors. Such a large mass is necessary to access the inverted hierarchy neutrino mass scheme, where $m_{3}<m_{1}<m_{2}$, in reasonable measuring time. One additional direction that has been followed in the COBRA study was the exploitation of CMOS-based hybrid active pixel detectors with a sensor layer of the II-VI-semiconductor $\mathrm{CdTe}$. The idea is that the topological structure of the tracks of the two decay electrons from a $0 \nu \beta \beta$-decay of ${ }^{116} \mathrm{Cd}$ might be used to discriminate between $0 \nu \beta \beta$-events and single electrons from background processes. Possible background processes include electrons from beta decays of radioactive impurities, Compton scattering of high-energetic photons caused by thermal neutron capture in any remaining ${ }^{114} \mathrm{Cd}$ impurities, or photon production after decays of radioactive impurities in the detectors.

Hybrid pixel detectors comprise an Application Specific Integrated Circuit (ASIC) realized in CMOS technology and a semiconductor sensor layer coupled pixel-wise to the ASIC by bump-bonding techniques. The ASIC is electronically segmented in both a matrix of pixels and a periphery for controlling and read-out of the pixel matrix. Hybrid pixel detectors have their origins in high-energy physics and are a very important part of the tracking systems in the LHC experiments. In ATLAS, CMS, and ALICE, tracking of highenergy charged particles is performed close to the vertex by detecting the energy depositions of charged particles in several layers of pixel detectors with silicon sensor layers. In LHCb, hybrid pixel detectors with silicon sensors are used to detect accelerated photoelectrons released from photocathodes by Cherenkov photons in the RICH detectors. The Timepix3 detector [16], which has just been developed by the Medipix collaboration, might be a promising option for a $\mathrm{LHCb}$ particle telescope to replace the current silicon microstrip system in the SLHC era. Can hybrid pixel detectors also help in neutrino physics experiments to decide whether the neutrino has Dirac or a Majorana character? By how much can one reduce the background with track analysis?

\section{Materials and Methods}

2.1. The Timepix Detector. The base of this study is the stateof-the-art hybrid active pixel detector Timepix [17] which is compatible with CdTe sensor layers. The Timepix detector has been developed by the Medipix collaboration [18] in the framework of the EUDET study. It is manufactured with the $0.25 \mu \mathrm{m}$ CMOS technology and features a $256 \times 256$ square 
matrix of electronic cells with $55 \mu \mathrm{m}$ pixel pitch. The area of the active matrix is $1.4 \times 1.4 \mathrm{~cm}^{2}$. Each of the electronics cells has a metallic input pad that can be connected by means of bump bonding to the electron collecting electrode of a CdTe layer.

It has already been demonstrated that $1 \mathrm{~mm}$ thick CdTe layers can be assembled with the Timepix ASIC $[19,20]$. Other hybrid pixel detectors like the HEXITEC detector already have been successfully contacted to even $3 \mathrm{~mm}$ thick CdZnTe sensors with $80 \times 80$ pixels at $250 \mu \mathrm{m}$ pixel pitch [21]. By extending the pixel electrodes of the CdTe layer laterally over several Timepix electronic pixel cells, one can realize sensor pixel sizes of a multiple of the active matrix pixel pitch $(55 \mu \mathrm{m})$. The average energy loss of an ionizing particle traveling through the sensor that is necessary to create an electron-hole pair is $4.43 \mathrm{eV}$ in CdTe. Positive and negative charge carriers are separated from each other by an electric field generated through a voltage difference between the common electrode and the pixel electrodes. The polarity of the voltage is chosen in a way that the electrons, having a larger mobility of $\mu_{e} \approx 1100 \mathrm{~cm}^{2} \mathrm{~V}^{-1} \mathrm{~s}^{-1}$ ) and mobility-lifetime product $(\mu \tau)_{e} \approx 3.0 \times 10^{-3} \mathrm{~cm}^{2} \mathrm{~V}^{-1}$ than holes $\left(\mu_{h} \approx\right.$ $\left.100 \mathrm{~cm}^{2} \mathrm{~V}^{-1} \mathrm{~s}^{-1},(\mu \tau)_{h} \approx 2.0 \times 10^{-4} \mathrm{~cm}^{2} \mathrm{~V}^{-1}\right)$ [22], drift towards the pixel electrodes. Both charge carrier types induce mirror charges on the pixel electrode plane. The induced current, caused by changes in the mirror charge in time due to the drift motions, is transferred to the input of the electronics pixel cell by the bump bond. In the Timepix electronics pixel cell this current pulse is amplified and converted to a voltage pulse with a peaking time of approximately $100 \mathrm{~ns}$ [23] (depending on the adjustable preamplifier gain) and a rather long falling edge with a duration in the microsecond range. The shaped pulse is input to a leading-edge discriminator that generates a gate to a counter. The minimum discriminator threshold that can be applied in conjunction with a silicon sensor with $55 \mu \mathrm{m}$ pixel pitch is about 900 electrons. In the case of a $1 \mathrm{~mm}$ thick CdTe sensor with $110 \mu \mathrm{m}$ pixel pitch, we were able to achieve a minimum threshold of 1185 electrons which corresponds to $5.5 \mathrm{keV}$.

The gate is open as long as the output pulse of the amplifier is above threshold (time-over-threshold mode) or extends until a global shutter signal inhibits the counter (time-ofarrival mode or time-of-detection mode). The counter counts the number of cycles of a clock signal supplied from the matrix periphery to each pixel as long as the gate is open. The maximum clock frequency that can be used in the Timepix is approximately $120 \mathrm{MHz}$. In time-over-threshold mode the counter value represents the length of the amplifier output pulse being above threshold. This duration is related to the induced current integrated over the shaping time which corresponds to the amount of charge collected by the pixel. Thus the time-over-threshold is a measure of the energy deposited by the ionizing particle in the sensitive volume of the pixel. In time-of-detection mode, the number of clock cycles measured in the counter of a pixel represents the moment of detection with respect to the moment of appearance of the shutter signal, which stops all counting activity. The moment of detection differs from the arrival time of the particle in the pixel. One reason is that a certain drift length has to be traversed until the influenced current integrated over the shaping time of the preamplifier exceeds the threshold level. The maximum drift time of charge carriers to the pixel electrodes is $73.5 \mathrm{~ns}$ for a $3 \mathrm{~mm}$ thick $\mathrm{CdTe}$ sensor biased at $-1500 \mathrm{~V}$. Additionally the time walk in the leading edge discriminator causes a delay of up to $100 \mathrm{~ns}$ depending on energy deposition and threshold level. Each pixel can also be operated in counting mode. The counter then counts the number of particles with energy depositions above threshold in the pixel during the shutter opening time.

Each pixel can be operated in exactly one of these three modes and thus deliver images of the number of detected events, images of energy depositions, or images of time stamps. The Timepix can in principle deliver all necessary information for $0 \nu \beta \beta$-decay detection: the topology of the particle track, the energy deposition in each pixel, and the timing of the event. The next version of the Timepix detector-the Timepix3 [16] - will provide energy information and timing information simultaneously in each pixel.

\subsection{The Detector Simulation}

2.2.1. Signal Generation. A Monte-Carlo simulation was developed for the precise simulation of electron tracks emerging from $0 \nu \beta \beta$-decays and for single electrons emerging from beta decays or Compton scattering. The event generator Decay0 [24] was used to generate the momenta of the two electrons emerging from $0 \nu \beta \beta$-decays distributed homogeneously in the CdTe layer. In the Monte-Carlo simulation framework ROSI [25], which is based on EGS4 with the lowenergy extension LSCAT, the tracks were sampled with steps of a few micrometers. Each track consisted of segments. A new segment was forced after an energy loss of $1 \%$ of the actual electron energy at most. We modeled the signal generation in the CdTe pixel detector as follows. The energy loss $\Delta E_{i}$ along a segment $i$ was converted to the corresponding number of generated electron-hole pairs $N_{i}=\Delta E_{i} /(4.43 \mathrm{eV})$ to calculate the contribution of the charge carriers released in segment $i$ to the induced current at the pixel electrode plane. The drift time was calculated for each segment $i$ taking the average distance of the track segment to the pixel electrode plane and the electric field strength into account. The electric field strength as a function of the distance from the pixel electrode $z$ was calculated for every combination of sensor thickness and bias voltage $U$ by $|\vec{E}|=|U| \cdot\left(f_{1} \cdot z+f_{2}+\right.$ $\left.f_{3} \cdot e^{-|U| \cdot f_{4} \cdot z}\right]$. We obtained $f_{1}=(5.80 \pm 0.09) \times 10^{5} \mathrm{~m}^{-2}$, $f_{2}=(228.0 \pm 7.5) \mathrm{m}^{-1}, f_{3}=(540 \pm 144) \mathrm{m}^{-1}$, and $f_{4}=(479 \pm$ 216) $\mathrm{V}^{-1} \mathrm{~m}^{-1}$ by fitting electric field strength measurements of various CdTe sensors. The exponential function accounts for the compression of electric field lines close to the pixel electrodes.

Guni et al. [27] have investigated charge sharing and energy resolution of CdTe sensors for various pixel sizes connected to the photon counting pixel detector Medipix2. Based on their study a Monte-Carlo simulation was developed that reproduced the energy deposition spectra for X-ray photons on the analog side of the pixel electronics [28]. In order to take charge carrier diffusion into account, the locations of the 
electrons arriving after drift at the pixel electrode plane were determined according to a Gaussian probability distribution depending on their drift time. The width of the Gaussian was calculated with the model presented by Spieler and Haller [29] adapted to the charge carrier transport properties of $\mathrm{CdTe}$. The charge induced by each drifting charge carrier in the pixel electrode was calculated using the Shockley-Ramo theorem $[30,31]$ which describes the calculation of currents induced in an electrode by a moving charge. The integral of the induced current over time is the induced charge $Q=$ $q \Delta \varphi$ where $\Delta \varphi$ is the difference of the weighting potential values of the starting point and the end point of the charge carrier trajectory and $q$ is the charge of the charge carrier. The weighting potential $\varphi(\vec{r})$ for a certain geometry of electrodes is the solution of the Laplace equation $\vec{\nabla}^{2} \varphi(\vec{r})=0$ with the boundary conditions that the weighting potential equals 1 at the sensing electrode and 0 at the positions of all other electrodes [32]. In the pixel detector the weighting potential is 1 at the pixel electrode (anode) and 0 at the common electrode (cathode). The weighting potential was calculated for each combination of sensor thickness and pixel pitch with the model presented by Castoldi et al. [26]. Figure 1 shows the weighting potentials for different combinations of thickness and pixel size. It can be seen that the largest portion of the signal is induced for electrons close to the pixel electrodes. In the simulation, the end-point of each charge carrier is determined by calculating what is reached first: the position after drift at the end of its lifetime, the electrode (anode for electrons or cathode for holes), or the end of the integration time in the preamplifier. To calculate the individual length of life of a charge carrier a random distribution according to the life-time of the charge carrier type was used. The total induced charge of an event-which is a measure of the collected energy deposition-was calculated as the sum of the induced charges of all carriers released along all segments of the track. The analog electronics noise and variations of discriminator threshold among pixels were taken into account by adding an energy deposition according to a Gaussian distribution with a standard deviation of 200 electron charges. In each pixel the simulated signal was compared to a discriminator threshold which was set to a realistic value of $7 \mathrm{keV}$ for the simulations of doublebeta decays and single electrons. If the energy was smaller than threshold, the signal in the pixel was ignored and not processed any further. In case the threshold was exceeded, a contribution $\Delta \mathrm{ToT}$ of the time-over-threshold noise was converted to energy and added to the collected energy in the pixel. Time-over-threshold noise is mainly due to voltage fluctuations on the falling edge of the preamplifier pulse when it comes close to the threshold.

2.2.2. Energy Resolution in the Pixel. To model time-overthreshold noise the energy-deposition $E$ in the pixel was first converted to a time-over-threshold value ToT with the calibration function $\operatorname{ToT}(E)=(a \times E+b)+c /(E-d) \cdot a$, $b, c$, and $d$ were derived for each pixel from measurements with X-ray fluorescence and radioactive sources. The first term in parentheses describes the linear dependence of ToT on the energy for deposited energies well above the threshold of the discriminator. This behavior is due to the triangular shape of the voltage pulse at the output of the preamplifier in the pixel electronics and due to the linearity of the preamplifier pulse height with collected charge. The exact shape of the tip of this pulse has strong influence on the time-over-threshold measurement if the pulse height is only slightly above discriminator threshold. The shape of the tip is the result of an interplay between the charge integration on the feedback capacitance and the discharging with a constant current in the preamplifier circuit. The linear relation between time-over-threshold and collected charge no longer holds for small amounts of collected charge: timeover-threshold becomes shorter than one would expect from the behavior at large amounts of collected charge. The second term in $\operatorname{ToT}(E)$ then becomes dominant. The applicability of this function to describe the energy measurements in timeover-threshold mode with the Timepix was first proved by Jakubek [33].

The standard deviation of time-over-threshold measurements was determined as a function of time-over-threshold by injecting test pulses into the input of the preamplifier of the Timepix. Randomizing a Gaussian distribution, centered at 0 , with this standard deviation gives a value $\Delta \mathrm{ToT}$ for the specific collected energy in the pixel. The sum ToT + $\Delta \mathrm{ToT}$ is then back-projected to the energy-deposition axis according to the above-mentioned calibration curve. Thus an energy-deposition value is obtained for each pixel in the simulation including electronics noise, time-over-threshold noise, electron and hole signal, limited charge carrier lifetime, weighting potentials, drift, and diffusion.

The full width at half maximum of the full energy peak $\Delta E_{\mathrm{FWHM}}$ of photoabsorption was measured by irradiation of the Timepix with X-ray fluorescence and with photons from radioactive decays. Figure 2 shows the relative energy resolution $\Delta E_{\mathrm{FWHM}} / E$ as a function of the detected energy $E$ for simulation and measurement. The relative energy resolution improves with increasing energy due to better accuracy of the time-over-threshold measurement. The timeover-threshold of pulses that are well above threshold for a longer time is affected (relatively) less by electronics noise on the falling edge of the pulse than for shorter pulses. The measured relative energy resolution $\Delta E_{\mathrm{FWHM}} / E$ in clusters of triggered pixels is $13.2 \%$ at $59 \mathrm{keV}, 8.2 \%$ at $80 \mathrm{keV}$, and $5.9 \%$ at $121 \mathrm{keV}$ detected energy. Good agreement is found for simulated and measured energy resolution values of the full pixel matrix after pixel-wise time-over-threshold calibration of the Timepix detector coupled to a $1 \mathrm{~mm}$ thick CdTe layer with $110 \mu \mathrm{m}$ pixel pitch in the X-ray energy range. The differences between simulated and measured values of the relative energy resolution were smaller than $0.6 \%$ for detected energies between $59 \mathrm{keV}$ and $121 \mathrm{keV}$. For lower energies the simulated energy resolution is worse than the energy resolution in the measurement. The total energy in all triggered pixels was taken as the measured energydeposition of the event if more than one pixel was triggered due to charge carrier diffusion or the track length of the photo- or Compton-electrons. Determination of $\Delta E_{\mathrm{FWHM}} / E$ for energies larger than about $121 \mathrm{keV}$ by irradiation with photons was almost impossible because in this energy range 


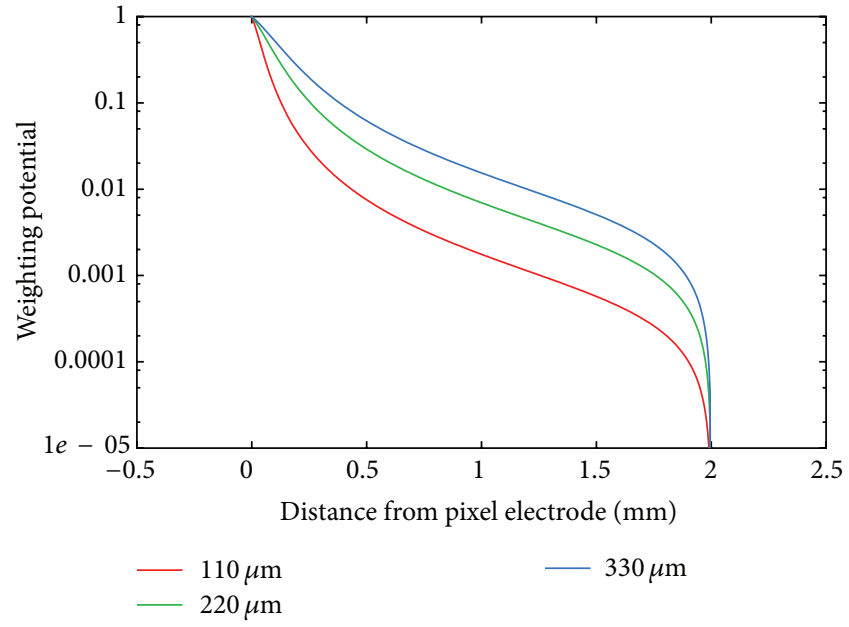

(a)

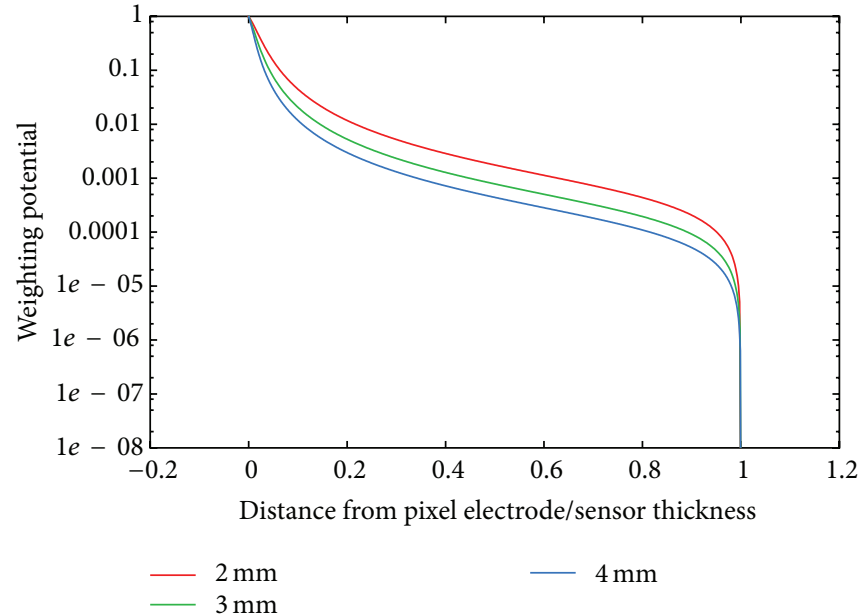

(b)

Figure 1: (a) Weighting potential for a $2 \mathrm{~mm}$ thick CdTe sensor for different pixel pitch as functions of the distance $z$ from the pixel electrode plane. (b) Weighting potential for different sensor thicknesses at fixed pixel pitch of $110 \mu \mathrm{m}$ as function of the ratio of the distance from the pixel electrode plane to the sensor thickness. Weighting potentials were calculated according to the model of Castoldi et al. [26].

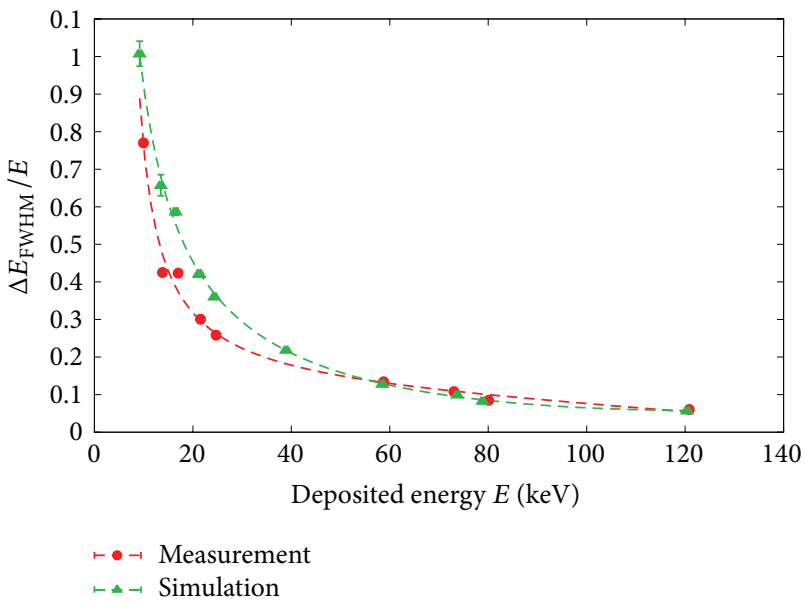

FIGURE 2: Simulated and measured relative energy resolution $\Delta E_{\mathrm{FWHM}} / E$ of the Timepix detector with a $1 \mathrm{~mm}$ thick CdTe sensor and $110 \mu \mathrm{m}$ pixel pitch in time-over-threshold mode as a function of deposited energy $E$. $\Delta E_{\mathrm{FWHM}}$ is the full width at half maximum of the full-energy peak at energy $E$.

the cross-section of the photoelectric effect strongly decreases with increasing photon energy.

2.2.3. Energy Resolution of Tracks. The question arises whether or not this energy resolution is sufficient for a $0 \nu \beta \beta$ decay search experiment. Figure 3 illustrates the doublebeta decay imaging chain of a $3 \mathrm{~mm}$ thick CdTe sensor with $110 \mu \mathrm{m}$ pitch. In Figure 3(a) the simulated "image" of the energy deposited in a $0 \nu \beta \beta$-event is shown. The color represents energy deposition in keV. In Figure 3(b) the image of the (energy-deposition equivalent) induced signals in the pixel electrodes is presented. Figure $3(\mathrm{~b})$ thus shows the appearance of the track in terms of input signal strength to the amplifier after drift, diffusion, trapping, and induction. Figure 3(c) shows the appearance of the event after in-pixel discrimination with a $7 \mathrm{keV}$ threshold. It looks similar but is not identical to the "real track" Figure 3(a).

An experiment has been carried out to test the simulation predictions of the energy resolution for electron tracks and to test a neural network for single-electron identification. The idea of the experiment was to produce two-chargedparticle track structures similar to $0 \nu \beta \beta$-decays in order to assess the energy resolution for the tracks. Electron-positron tracks were generated by pair production. The experiment and the results were presented by Filipenko et al. [20] in more detail. A $1 \mathrm{~mm}$ thick CdTe sensor layer with ohmic contacts (Pt) biased continuously at $-500 \mathrm{~V}$ was exposed from the side to $2.614 \mathrm{MeV}$ photons from the decay of ${ }^{208} \mathrm{Tl}$. The Timepix detector used in this study was assembled by the Freiburger Materialforschungszentrum FMF which has also evaluated CdTe as sensor material on Timepix detectors [19]. The setup was not temperature stabilized. Due to the heat dissipation from the ASIC the temperature of the CdTe sensor was approximately $37^{\circ} \mathrm{C}$. Stabilization of the detector at lower temperature is an option for leakage current reduction and possibly for an improvement of the energy resolution. Images in time-over-threshold mode were taken using the USB readout [34] controlled by the data acquisition software Pixelman [35]. The probability for pair production is $16.2 \%$ of the Compton scattering probability in $\mathrm{CdTe}$ at this photon energy. Thus, the spectrum of energies in tracks was dominated by Compton scattering. The images have been analyzed for single-electron tracks and electron-positron track pairs with the artificial neural network FANN [36]. The network comprised 10 input units and 1 output unit on 5 hidden layers with 200 neurons each. The activation function $\tanh (0.3 x)$ with the learning method Resilient Propagation produced the best results. The neural network was trained with simulated tracks and was applied 


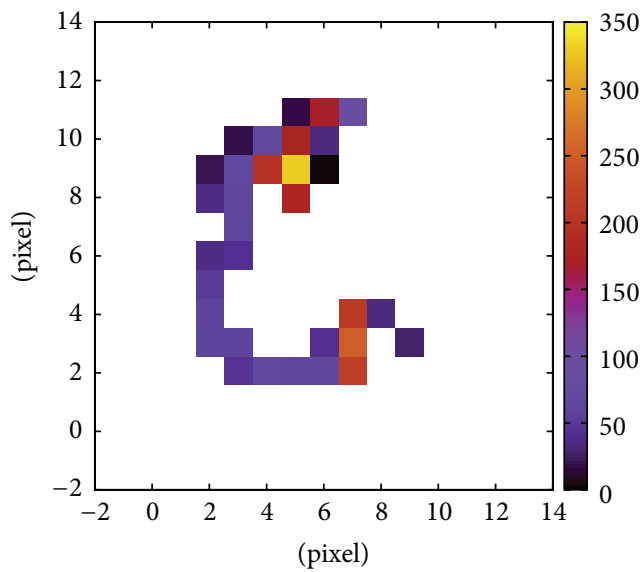

(a) Deposited energy in the geometric volume of each pixel

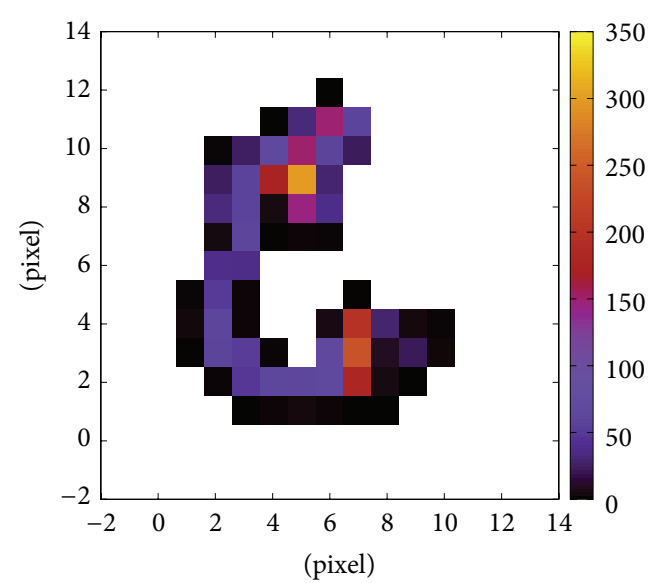

(b) The input to the amplifier in each pixel

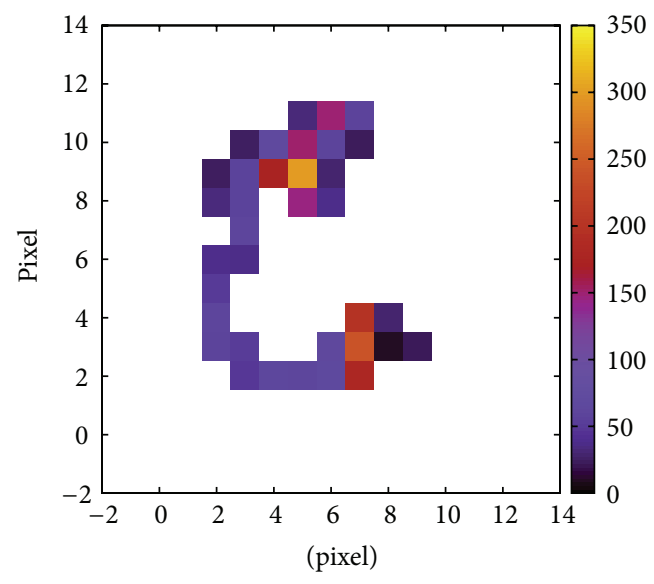

(c) After discrimination

FIGURE 3: Example of the appearance of a $0 \nu \beta \beta$-track, simulated for a $3 \mathrm{~mm}$ thick CdTe sensor with a pixel pitch of $110 \mu \mathrm{m}$, at different stages of the imaging chain. The $x$ - and $y$-coordinates give the pixel row and column. The color bar gives the deposited or measured (time-overthreshold) energy in keV.

to measured data. The sum of the energy depositions in all pixels in the tracks has been determined for each track structure after removing $\alpha$-particle signatures and muon tracks. Figure 4 shows the measured spectra of deposited energies in tracks for pair production and single-electron events after reconstruction with the neural network where the known classification errors of the network have been corrected for. A clear peak from pair production is visible. The corrected spectrum for single-electron tracks almost shows no relict of a pair-production peak. The energy resolution in the pair production electron-positron tracks was measured to be $\Delta E_{\mathrm{FWHM}} / E=3.77 \%$, which was about twice the resolution that was expected from simulation. This discrepancy was due to the extrapolation of the time-over-threshold versus energy calibration curves from the highest photon energy used for calibration $(121 \mathrm{keV})$ to deposited energies of up to $400 \mathrm{keV}$ in single pixels. High-energy photons of several hundred $\mathrm{keV}$ cannot be used for energy calibration because of the small cross-section of the photoelectric effect and the long electron tracks at these energies. Photoelectrons of these energies are mostly not confined in one pixel so that for each calibration event the energy depositions in individual pixels would be unknown. Nevertheless, the results of the experiment showed that the simulation and the track analysis with the neural network worked well since the separation between singleelectron tracks (blue in Figure 4) and pair-production events (green in Figure 4) was as expected.

\section{Results and Discussion}

3.1. Simulation Setup. All simulations of double-beta decays were carried out with a geometry where $2 \times 2$ Timepix detectors with CdTe sensors are packed together in contact to each other. Two of such $2 \times 2$ matrices were placed on top of each other with their CdTe sensors facing each other. With this arrangement the loss of electrons at the edges was reduced. Furthermore, electrons which leave the CdTe sensors at the common electrodes could deposit their remaining energy in the opposite $\mathrm{CdTe}$ detector. This increases the $0 \nu \beta \beta$ detection efficiency.

Test measurements [20] have shown that a bias voltage of $-500 \mathrm{~V}$ was an appropriate choice for the $1 \mathrm{~mm}$ thick 


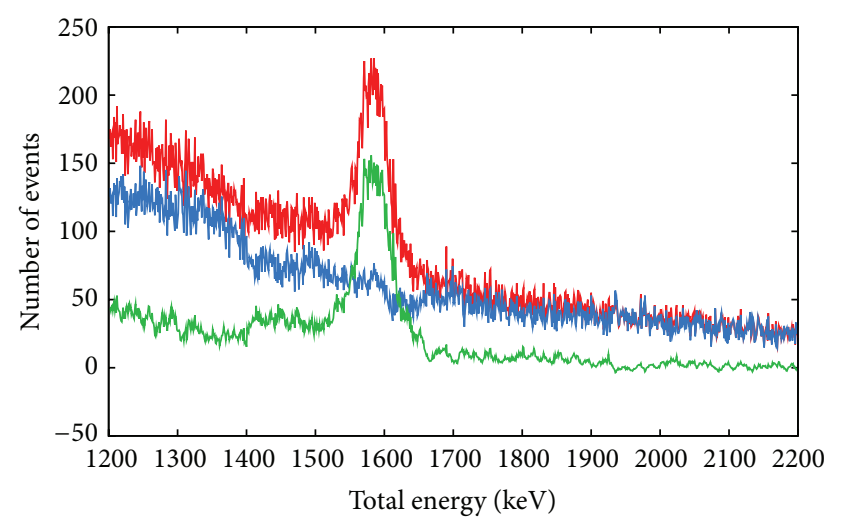

Measurement
- Single electrons reconstructed
Pair production reconstructed

FIGURE 4: Red: reconstructed distribution of measured total energy depositions in tracks of a $1 \mathrm{~mm}$ thick CdTe Timepix detector with $110 \mu \mathrm{m}$ pixel pitch for irradiation with $2.614 \mathrm{MeV}$ photons through its edge. Straight line tracks caused by muons and circular track structures caused by $\alpha$-particles have been removed before track analysis with the artificial neural network. Green: reconstructed total-energy distribution for events that should stem from pair production. Blue: reconstructed total-energy distribution of singleelectron tracks.

sensor with ohmic contacts. The drift times were reasonably short: the blurring of tracks due to diffusion of charge carriers was not too strong. The leakage current of $0.8 \mathrm{nA}$ per pixel at a sensor temperature of approximately $37^{\circ} \mathrm{C}$ was at an acceptable level so that a reasonably low discriminator threshold of $5.5 \mathrm{keV}$ could be realized experimentally with $110 \mu \mathrm{m}$ pixel pitch. The leakage current compensation circuit in each pixel of the Timepix can even compensate for leakage currents up to $-10 \mathrm{nA}[23]$ in electron collection mode. Even with $-700 \mathrm{~V}$ bias voltage only $1.3 \%$ of the pixels did not work properly [20]. It shall be pointed out that in principle the leakage current could be further reduced either with Schottky contacts instead of ohmic contacts on the CdTe layer [21] or by cooling the detector which would probably also improve energy resolution. Remoué et al. [37] demonstrated that the leakage current of $4 \times 4 \times 1 \mathrm{~mm}^{3}$ CdTe sensors with indium Schottky contacts operated at $-20^{\circ} \mathrm{C}$ with $-600 \mathrm{~V}$ bias voltage can be smaller than $100 \mathrm{pA}$. This value would correspond to only $1.9 \mathrm{pA}$ leakage current per pixel for $550 \mu \mathrm{m}$ pixel pitch. The analog pixel electronics of the Timepix ASIC can even compensate for three orders of magnitude stronger leakage currents. The drawback of Schottky contacts would be that the sensor would tend to polarize [21] due to the buildup of space charges. Polarization in CdTe typically worsens charge collection efficiency and can be reduced by cooling [38]. The influence of the polarization effect could also be overcome by switching off the bias voltage for some seconds every few minutes [39]. It has already been shown [40] that almost complete charge collection can be obtained in $0.5 \mathrm{~mm}$ thick CdTe and $2 \mathrm{~mm}$ thick CZT layers with low bias voltages of about $-100 \mathrm{~V}$. Therefore, we assumed for our simulations of the performance of a pixel detector for various combinations

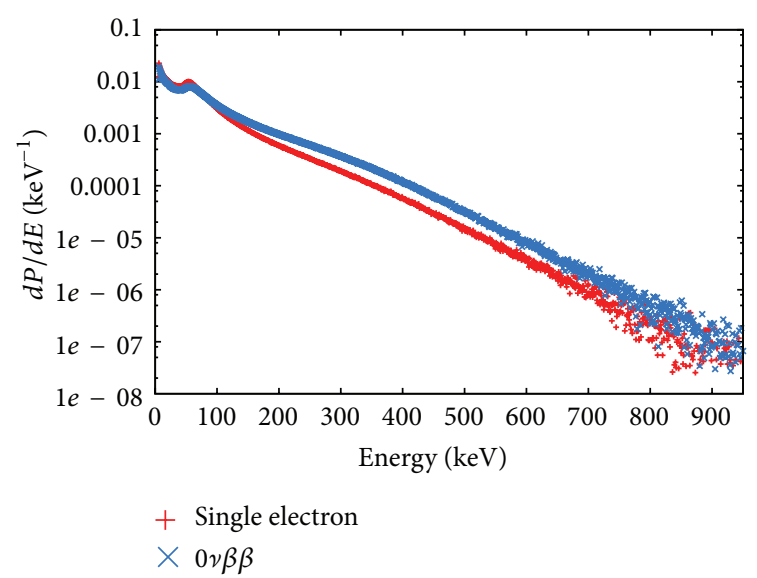

FIgure 5: Probability density $d P / d E$ that a triggered pixel in a track has collected a certain energy $E$ for neutrinoless double-beta decay (blue) and for single electrons (red). 2.8 MeV total deposited energy in the track was required for both event types.

of pixel pitch and sensor thickness that the pixel detector can be operated with bias voltages of $-500 \mathrm{~V}$ per mm sensor thickness.

The energy resolution of a single pixel is $\approx 13 \%$ (FWHM) for $\approx 59 \mathrm{keV}$ energy deposition. The question arises whether or not a sufficient energy resolution for $0 \nu \beta \beta$-decay detection can be obtained with this kind of detector.

\subsection{Total-Energy Spectra of Double-Beta Decay Events.} Figure 5 shows the simulated spectrum of detected energies in individual pixels caused by $0 \nu \beta \beta$-decays and by single electrons for $2.8 \mathrm{MeV}$ energy deposition in all triggered pixels of an event. The spectra were calculated for a $3 \mathrm{~mm}$ thick CdTe sensor with $110 \mu \mathrm{m}$ pixel pitch. For $0 \nu \beta \beta$-events most of the pixels record energies below $100 \mathrm{keV}$ and some pixels could receive energy deposition of more than $700 \mathrm{keV}$. In single-electron events, pixels are triggered more likely with smaller energy-depositions due to the lower ionization density for more energetic electrons. Larger energy depositions occur with a higher probability in $0 \nu \beta \beta$-events. A large fraction of pixels is triggered by small amounts of energy. At low energies the energy resolution of the Timepix detector with its Wilkinson type ADC (time-over-threshold) is not the best. We therefore studied whether or not this hybrid pixel detector with time-over-threshold measurement could achieve sufficient energy resolution for the two-electron track structure to obtain sufficient separation between the peak expected at the full $Q$-value of the decay (2813.50 \pm $0.13) \mathrm{keV}$ [13] and the high-energy tail of the unavoidable $2 v \beta \beta$-background. The pixel pitch and the thickness of the CdTe sensor layer were the most important free parameters in the design of the pixel detector.

Figure 6 shows the spectra of total detected energy for $0 \nu \beta \beta$-decay in comparison to the spectrum of the two electrons emitted in $2 \nu \beta \beta$-decays in the region of the $Q$-value for four different combinations of pixel size and thickness of the CdTe layer. An effective Majorana neutrino mass of $42 \mathrm{meV}\left(T_{1 / 2}^{0 v}=2.6 \times 10^{26} \mathrm{yr}\right)$ and a half-life of 


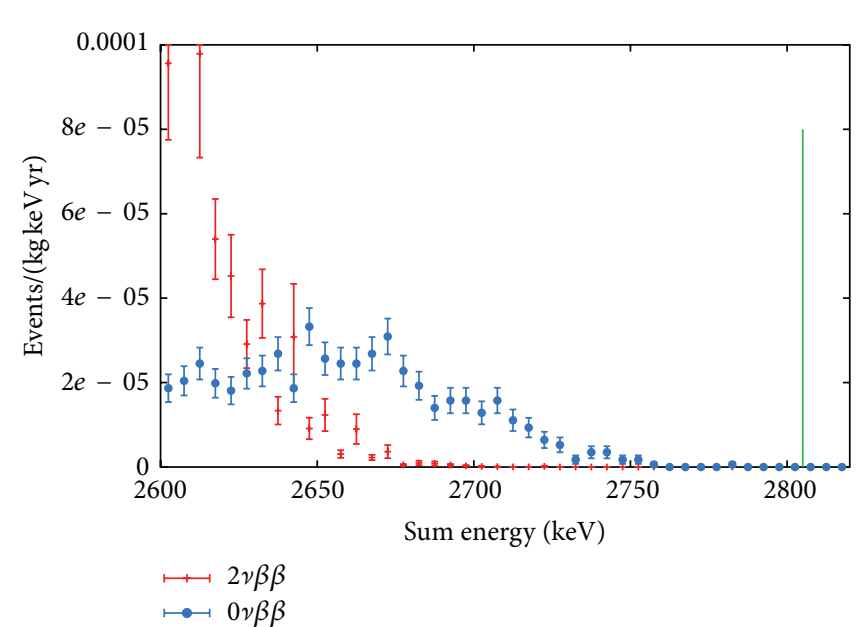

(a) $2 \mathrm{~mm}$ thickness, $55 \mu \mathrm{m}$ pitch

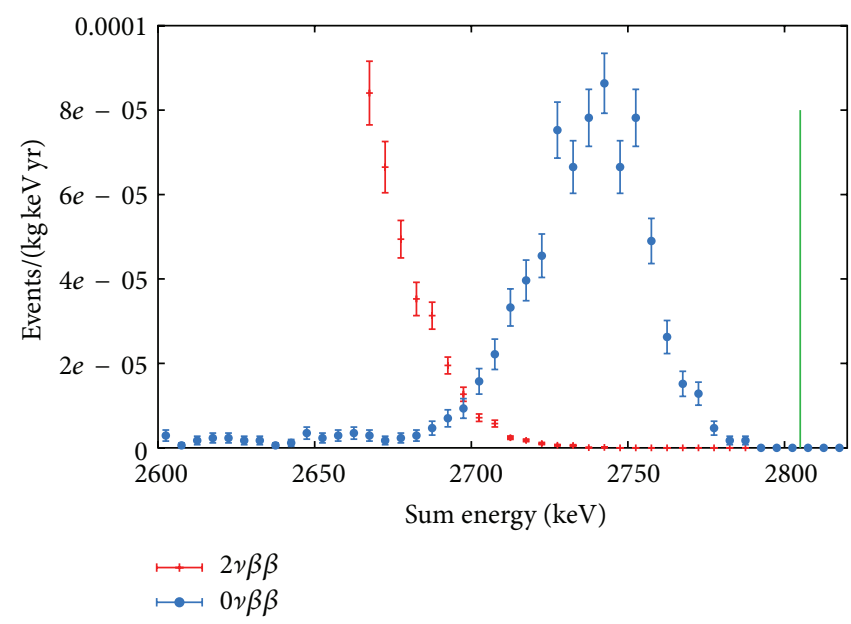

(c) $3 \mathrm{~mm}$ thickness, $165 \mu \mathrm{m}$ pitch

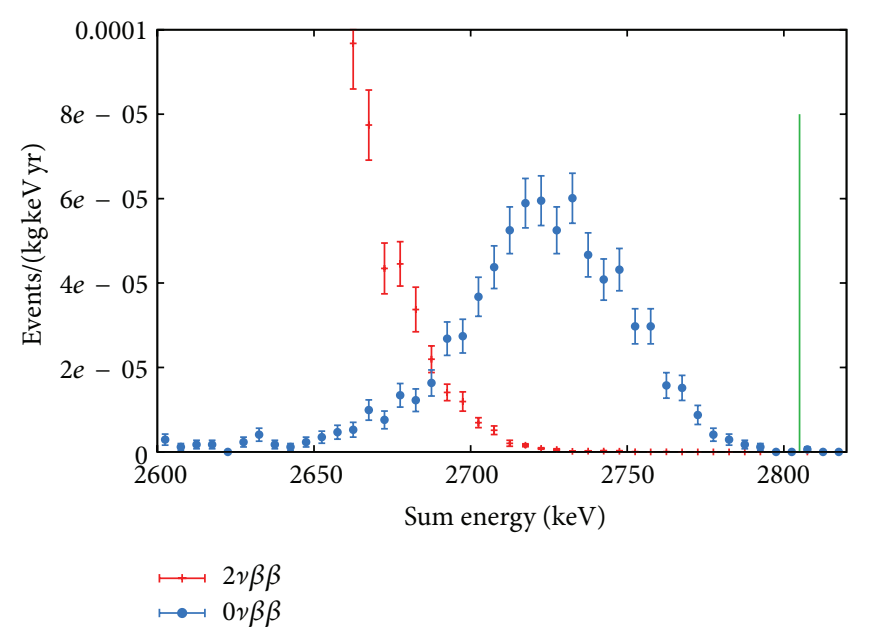

(b) $3 \mathrm{~mm}$ thickness, $110 \mu \mathrm{m}$ pitch

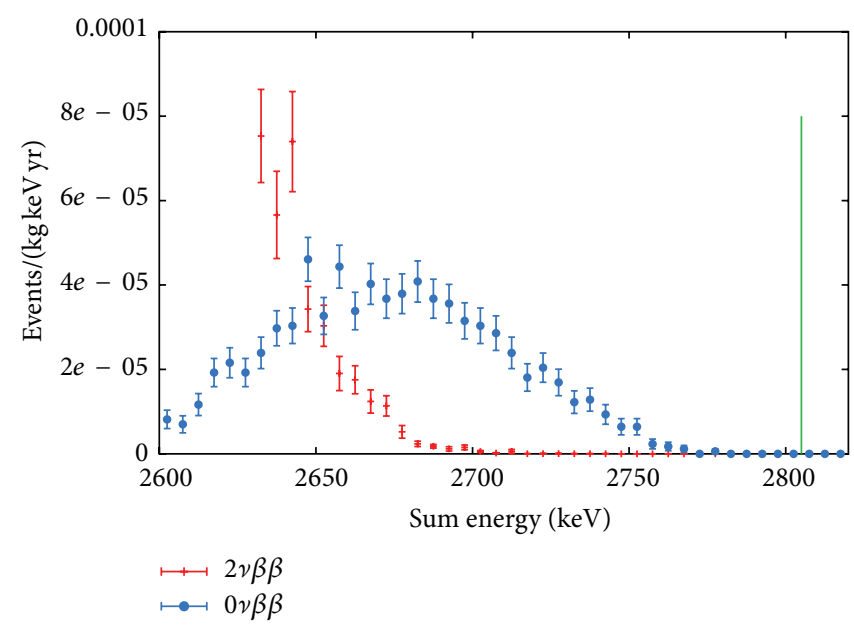

(d) $5 \mathrm{~mm}$ thickness, $110 \mu \mathrm{m}$ pitch

FIGURE 6: Simulated spectra of the total energy given in number of events per $\mathrm{kg} \times \mathrm{keV} \times \mathrm{yr}$ for $0 \nu \beta \beta$ - (blue) and $2 \nu \beta \beta$-decays (red). Cadmium was assumed to be enriched to $90 \%$ in ${ }^{116} \mathrm{Cd}$. The green vertical line indicates the position of the $Q$-value of the decay. The shifts of the peak positions for $0 \nu \beta \beta$-decays to deposited energies smaller than the $Q$-value is due to charge sharing at the edges of the electron tracks.

$T_{1 / 2}^{2 \nu}=2.88 \times 10^{19} \mathrm{yr}[14]$ were assumed. The simulation of the $2 \nu \beta \beta$-spectra was carried out separately in intervals of the sum of emission energies of the two electrons. The reason is that the probability for a $2 \nu \beta \beta$-decay decreases with increasing sum of the energies of the two electrons in the region of the $Q$-value. The numbers of detected events as a function of the total detected energy were weighted according to the probability that a $2 \nu \beta \beta$-decay occurs with the sum of energies of the two electrons in the interval. This results in statistic errors in the simulated $2 \nu \beta \beta$-spectra in Figure 6 which decrease with improving energy resolution.

We observed that the signal peaks did not appear at the $Q$-value of $2.814 \mathrm{MeV}$ but at a smaller energy $E_{Q}=Q-\Delta E_{\text {shift }}$ depending on bias voltage, pixel size, and sensor thickness. This loss of total energy is mainly due to the loss of charge from each triggered pixel to under-threshold neighboring pixels. The loss of charge is mainly due to diffusion of charge carriers during drift towards the pixel electrodes. The average energy loss $\Delta E_{\text {shift }}$ in the signal is about $70 \mathrm{keV}$ for the configuration with best energy resolution. The distance between the signal peak and the $Q$-value increases with increasing sensor thickness, decreasing bias voltage, and decreasing pixel size. The same effect shifts the spectrum of electron energies from $2 \nu \beta \beta$-decays towards lower energies. For a $3 \mathrm{~mm}$ thick sensor layer with $165 \mu \mathrm{m}$ pixel pitch the contribution of the $2 v \beta \beta$-decay channel to the number of accepted events would be only about $1.6 \%$. Here, we assumed an effective Majorana neutrino mass of $m_{\beta \beta}=42 \mathrm{meV}$ and an acceptance window width for signal events of $\Delta E_{\text {cut }}=59 \mathrm{keV}$ centered at $2738 \mathrm{keV}$.

3.3. Total-Energy Resolution. 2000 neutrinoless double-beta decay events with random electron momenta and positions in the pixel matrix were simulated to determine the energy resolution for each combination of pixel pitch and sensor layer thickness. Figure 7 shows the energy resolution $\Delta E_{\mathrm{FWHM}}\left(E_{\mathrm{Q}}\right) / E_{\mathrm{Q}}$ of the two-electron track signature at $E_{\mathrm{Q}}$ as functions of the pixel pitch for different values of the 


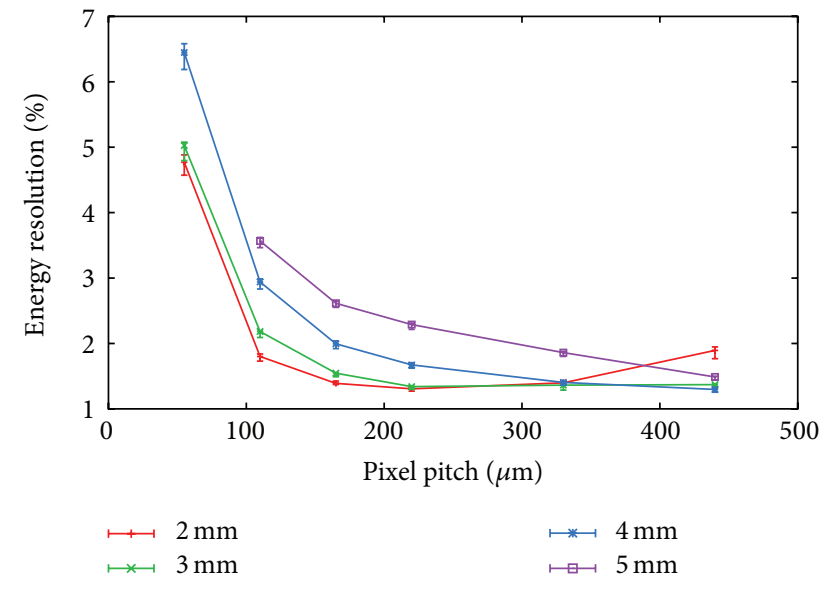

FigURE 7: Simulated relative energy resolution $\Delta E_{\mathrm{FWHM}}\left(E_{\mathrm{Q}}\right) / E_{\mathrm{Q}}$ for various combinations of pixel pitch and sensor thickness.

sensor thickness for bias voltages of $-500 \mathrm{~V}$ per mm sensor thickness. Such good energy resolutions can be achieved because the errors made in each pixel by the time-overthreshold measurement average out to a certain extent in the track. Total-energy resolution of the tracks improves with decreasing pixel pitch (increasing number of triggered pixels) unless the losses of energy underneath discriminator thresholds become significant. If smaller pixels are used, the number of pixels at the edge of the track is larger and the loss of charges to untriggered pixels adjacent to the triggered pixels becomes larger. The collected energy then depends strongly on the exact trajectories with respect to the borders of the pixels. Thus, the energy resolution for the tracks is worse if pixels are too small. A similar argument can be applied if the sensor is too thick.

For $0 \nu \beta \beta$-tracks the best energy resolution of about $\Delta E_{\mathrm{FWHM}}\left(E_{\mathrm{Q}}\right) / E_{\mathrm{Q}}=1.31 \%$ in the sum electron energy was achieved with a $2 \mathrm{~mm}$ thick CdTe sensor layer with $220 \mu \mathrm{m}$ pixel size at a bias voltage of $-500 \mathrm{~V}$ per mm sensor thickness. The sensor thickness, pixel size combinations $((3 \mathrm{~mm}, 330 \mu \mathrm{m})$ and $(4 \mathrm{~mm}, 440 \mu \mathrm{m}))$ showed only slightly worse energy resolutions.

3.4. Sensor Thickness. So far, the detection efficiency has not been considered. Thicker sensor layers have the advantage that less pixels are necessary to reach a certain mass of CdTe and therefore a certain sensitivity to the effective Majorana neutrino mass. A thicker sensor has several other advantages: increased volume-to-surface ratio and thus reduced specific background rate from surfaces, reduced total static power consumption of the analog pixel electronic cells, reduced necessary number of events for pixel calibration, and reduced bump-bonding costs. The fraction of the tracks that are fully contained in the sensor increases with sensor thickness. On the other hand, energy resolution for tracks eventually worsens with increasing sensor thickness because of the increasing influence of charge splitting (charge sharing) between pixels. Figure 8 presents the simulated half-life sensitivity according to the unified approach-which is presented in [41] — for a confidence level of $90 \%$ after 5 years of measuring time with

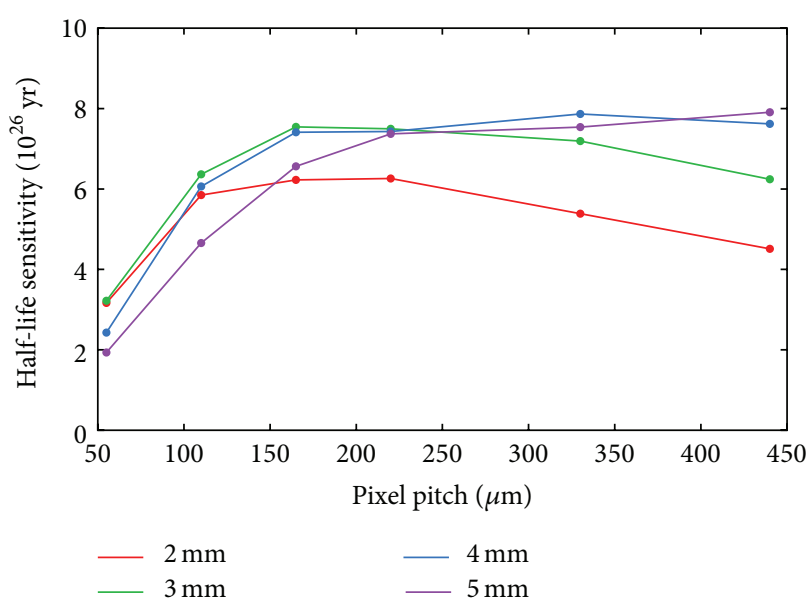

Figure 8: Simulated half-life sensitivity of a $420 \mathrm{~kg}$ CdTe pixel detector experiment (Cd enriched to $90 \%$ in ${ }^{116} \mathrm{Cd}$ ) for various combinations of pixel pitch and sensor thickness. Background sources other than $2 \nu \beta \beta$-decays were neglected at this stage.

$420 \mathrm{~kg} \mathrm{CdTe}\left(90 \%{ }^{116} \mathrm{Cd}\right)$ in the face-to-face setup of pixel detectors. In terms of sensitivity the best choice would be a sensor which is thicker than $2 \mathrm{~mm}$, although the best totalenergy resolution was found for the configuration $(2 \mathrm{~mm}$, $220 \mu \mathrm{m})$. At this stage of the analysis, the best sensitivities were obtained with a $4 \mathrm{~mm}$ thick sensor with $330 \mu \mathrm{m}$ pixel size or with a $5 \mathrm{~mm}$ thich sensor with $440 \mu \mathrm{m}$ pixel size. The simulated half-life sensitivity for $3 \mathrm{~mm}$ thickness and $165 \mu \mathrm{m}$ pixel size is very close to the half-life sensitivities of the configurations $((4 \mathrm{~mm}, 330 \mu \mathrm{m})$ and $(5 \mathrm{~mm}, 440 \mu \mathrm{m}))$. It can also be seen that the simulated half-life sensitivity does not strongly depend on the pixel size for $3 \mathrm{~mm}$ thickness between $165 \mu \mathrm{m}$ and $220 \mu \mathrm{m}$. For calculation of the sensitivities in Figure 8 only background from $2 \nu \beta \beta$-decays was taken into account in the region of $E_{Q}$. In an experiment, background like electrons from the decay of ${ }^{214} \mathrm{Bi}$, Compton scattered electrons, and $\alpha$-particles would also be present. It can be expected that discrimination of signal and single-electron background by analysis of the track topology is more effective with smaller pixel sizes. Track resolution is worse with thicker sensor layers because of charge sharing. Thicker sensors and a larger pixel pixel thus complicate the background rejection with track analysis. Therefore, the combination of a $3 \mathrm{~mm}$ CdTe layer thickness with a pixel size of $165 \mu \mathrm{m}$ seems to be the optimal compromise for a pixel detector. The detection efficiency for $0 \nu \beta \beta$-decay events with this optimal configuration is $55.9 \%$.

3.5. $0 \nu \beta \beta$ - and Single-Electron Tracks. The main motivation for employing pixel detectors in the search for neutrinoless double-beta decay is their ability to visualize tracks. Track analysis shall be used to discriminate background events. For example, electrons or positrons of sufficient energy can emerge from beta decays from radioactive contaminants either present in the CdTe sensor, the bump-bond material, the electrode metals, the ASIC, the readout electronics close to the CdTe sensors, the shielding, or the support 
structures. ${ }^{214} \mathrm{Bi}$, for example, undergoes beta decay with a $Q$-value of 3.3 MeV which is above the $Q$-value of the doublebeta decay of ${ }^{116} \mathrm{Cd}$. High-energy background electrons can also be released by Compton scattering in the sensor or surrounding parts by high-energy photons. Such photons can be generated, for example, in $\gamma-\gamma$-cascades after thermal neutron capture ${ }^{113} \mathrm{Cd}(n, \gamma){ }^{114} \mathrm{Cd}$ of ${ }^{113} \mathrm{Cd}$ remnants with a very large cross-section.

Figure 9 shows the appearance of an example of a $0 \nu \beta \beta$ decay event in pixel matrices with differing pitch ranging from $55 \mu \mathrm{m}$ to $880 \mu \mathrm{m}$ to illustrate the influence of pixel pitch on track resolution.

Figure 10 shows examples of images of simulated energy depositions in the pixel matrix for "obvious" Figure 10(a) and "deformed" Figure 10(b) $0 \nu \beta \beta$-events for a $3 \mathrm{~mm}$ thick CdTe sensor with a pixel pitch of $110 \mu \mathrm{m}$ at a discriminator threshold of $7 \mathrm{keV}$. A bias voltage of $-1500 \mathrm{~V}$ was applied. The appearance of the tracks in the image is affected by the diffusion of charge carriers during their drift, electron scattering in the CdTe sensor, and noise in the electronics. The naive picture is that a double-beta decay appears as two regions with large energy deposition per pixel (Bragg peak) that are connected by a thinner line of pixels. One can see in Figure 10(b) that this is not always the case. The main reason is that due to scattering off lattice atoms, the electrons can also move parallel to the electric field lines. They then deposit a large amount of energy in a few neighboring pixels, which looks like a Bragg-peak region.

Figure 11 shows two examples of simulated track images of single electrons emerging, for example, from a beta decay of ${ }^{214} \mathrm{Bi}$ contaminants with a total energy detected by the pixel matrix close to $E_{\mathrm{Q}}$. Comparing these images with the images of signal events presented in Figure 10, it is obvious that some of the single-electron tracks will look like the two tracks of two electrons from double-beta decay and vice versa.

3.6. Discrimination of Single-Electron Background. We used random decision forests for discrimination between singleelectron tracks and double-beta decay event pattern. The concept of random decision forests is based on the class of decision tree algorithms which are widely used for data classification. The basic idea of random decision forests has been described by Ho [42]. Random decision forests typically have an increased ability to generalize. They preserve the advantages of decision trees like a high classification performance and a very fast execution. Random decision forests use random feature subspaces to construct multiple decision trees which carry out a majority vote. The implementation of the random decision forest used in this study is based on the library ALGLIB [43]. The classification program was trained with simulated electron tracks of double-beta decay and with single electrons, both causing a measured energy close to $E_{\mathrm{Q}}$ in the pixel matrix. The cut in the decision process for identifying an event as signal or background is optimized for the best sensitivity of the experiment to the effective Majorana neutrino mass. In the following we describe in detail the 10 features that were used in the event classification. In Figures 12 and 13 we show the probability for obtaining a feature value in a certain bin width obtained with simulations of
$0 v \beta \beta$-decay events and single electrons with a total energy deposition in an acceptance window of $59 \mathrm{keV}$ width around $E_{\mathrm{Q}}$ for a $3 \mathrm{~mm}$ thick CdTe sensor with $110 \mu \mathrm{m}$ pixel pitch at a bias voltage of $-1500 \mathrm{~V}$.

For $0 \nu \beta \beta$-decay events we expect less pixels to be triggered on average than for a single electron at the same total energy. Thus, the number of triggered pixels (Figure 12(a)) in the track should give a hint whether a track structure is caused by a signal or background event.

A single electron should lose less energy per pixel at the beginning of its track and trigger many pixels in a small region when its energy comes close to the Bragg-peak region where the collected energy per pixel should be larger. Therefore, we expect that the center of gravity of the energy deposition pattern is different from the center of gravity of the binary track structure. In an "ideal" double-beta decay we would expect to see a pattern of two single-electron tracks connected in the pixel with the decaying nucleus. We expect a smaller distance between the energy-weighted and binary center-of-gravity of the track structure than for a single electron. The distance $d \equiv \sqrt{\left(\vec{r}_{\text {weighted }}-\vec{r}_{\text {binary }}\right)^{2}}$ (Figure 12(b)) becomes a feature of the classification process, where $\vec{r}_{\text {weighted }}$ is the center of gravity of the energy deposition in the track structure and $\vec{r}_{\text {binary }}$ is the center of gravity of the pattern of triggered pixels.

The beginning of a single-electron track shouldideally-begin with a thin, short structure of triggered pixels with relatively low energy deposition per pixel. In ideal double-beta decay events, such a structure should not be present at the ends of the track structure. We introduced two features in the classification process: the total energy measured in two (Figure 12(c)) or in three (Figure 12(d)) adjacent pixels with only one neighboring triggered pixel. The smallest of these energy values is used if several of these structures exist in an event. We defined that neighboring pixels must touch each other and be located either in the same row or in the same column.

As additional features we used the length of a structure at the edge of the track in which each pixel has only one triggered neighbor, which in turn had only one triggered neighbor and so on. Typically these structures are longer for single-electron tracks (Figure 12(e)).

For $0 \nu \beta \beta$-decay events we ideally expect to see two distant structures with high energy depositions per pixel (e.g., > $250 \mathrm{keV}$ ), because of Bethe-Bloch-like energy losses. Thus, we defined the maximum distance of pixels in the track having detected energies of more than $250 \mathrm{keV}$ as a feature (Figure 12(f)).

A single background electron with a total energy deposition in the region of $E_{Q}$ should deposit less energy per pixel than an electron from a $0 \nu \beta \beta$-decay because of its higher energy. The number of pixels having measured a total energy of less than $185 \mathrm{keV}$ together with their neighbors became a feature (Figure 13(a)) that gives on average larger values for single electrons.

We expect ideal single-electron tracks to have a line of triggered pixels at the beginning of the track. Therefore, the 


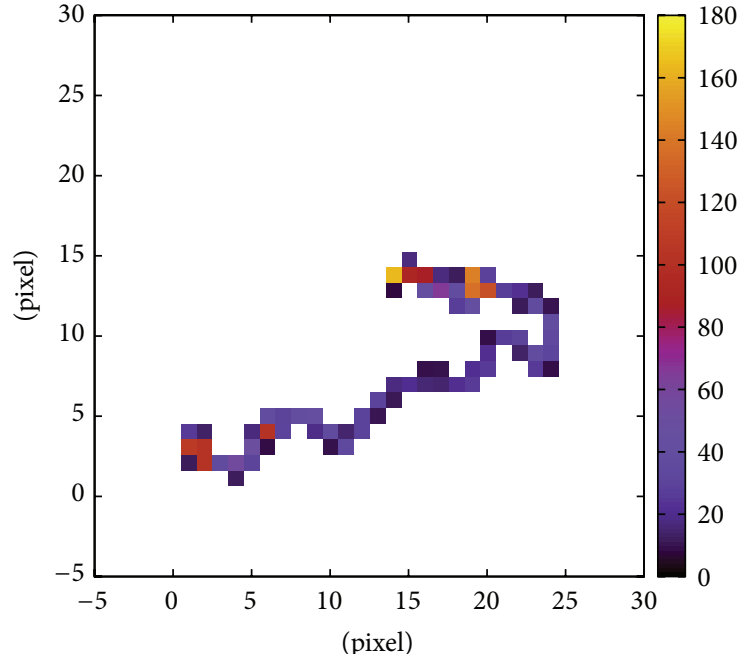

(a) $55 \mu \mathrm{m}$

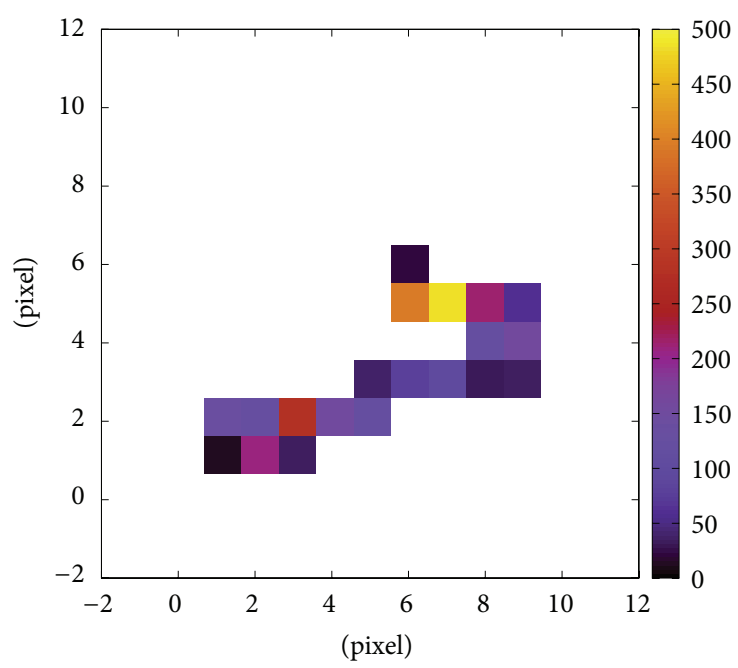

(c) $165 \mu \mathrm{m}$

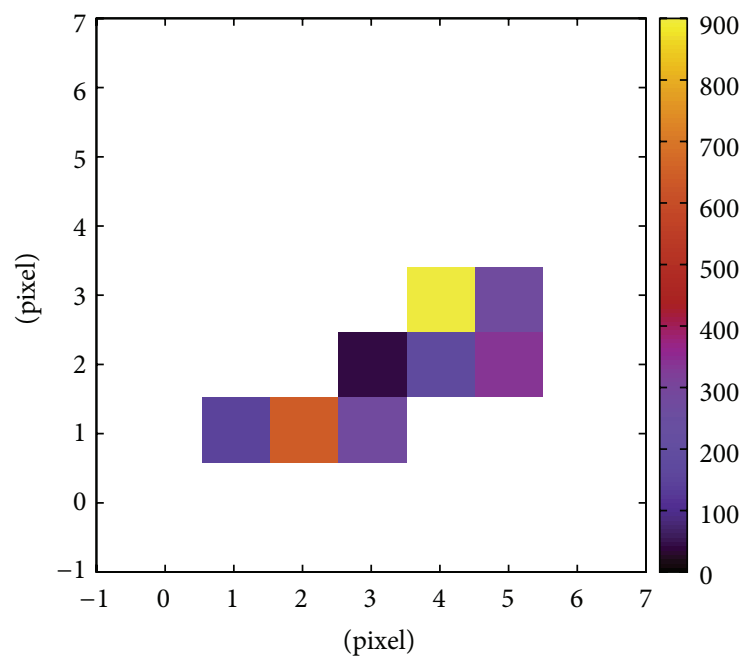

(e) $330 \mu \mathrm{m}$

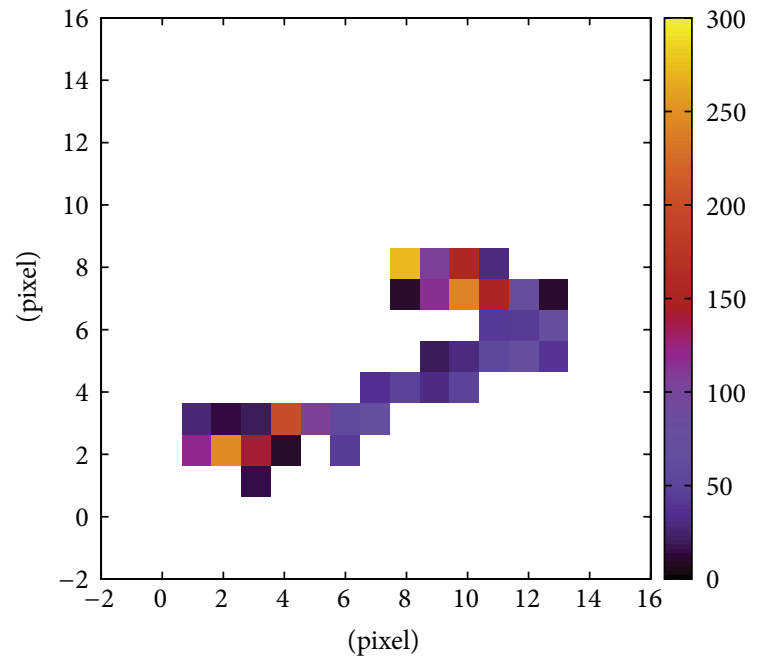

(b) $110 \mu \mathrm{m}$

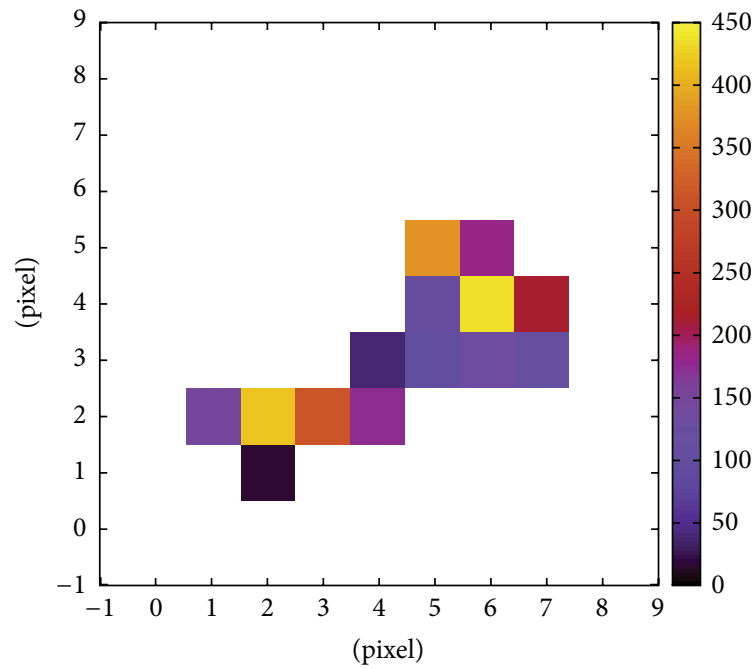

(d) $220 \mu \mathrm{m}$

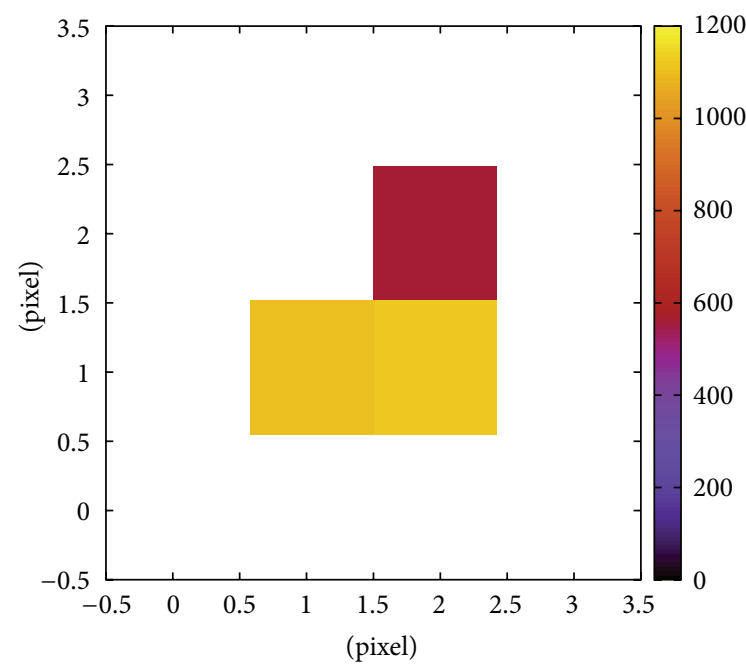

(f) $880 \mu \mathrm{m}$

FIGURE 9: Simulated pattern of energy depositions in the pixel matrix for a $0 \nu \beta \beta$-decay event in a 3 mm thick CdTe sensor for different granularities of the pixel matrix. The $x$ - and $y$-coordinates give the pixel row and column. The color bar encodes the energy deposition measured with the time-over-threshold method in $\mathrm{keV}$. 


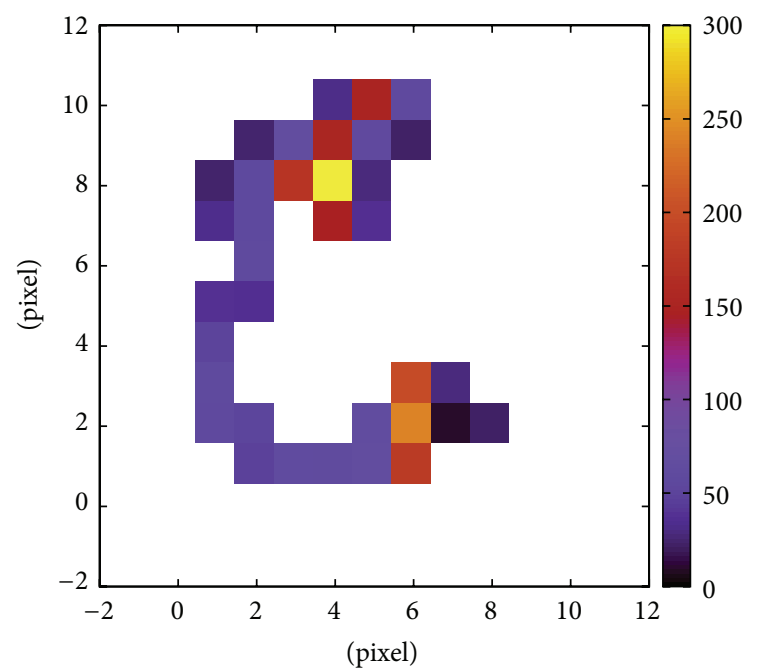

(a)

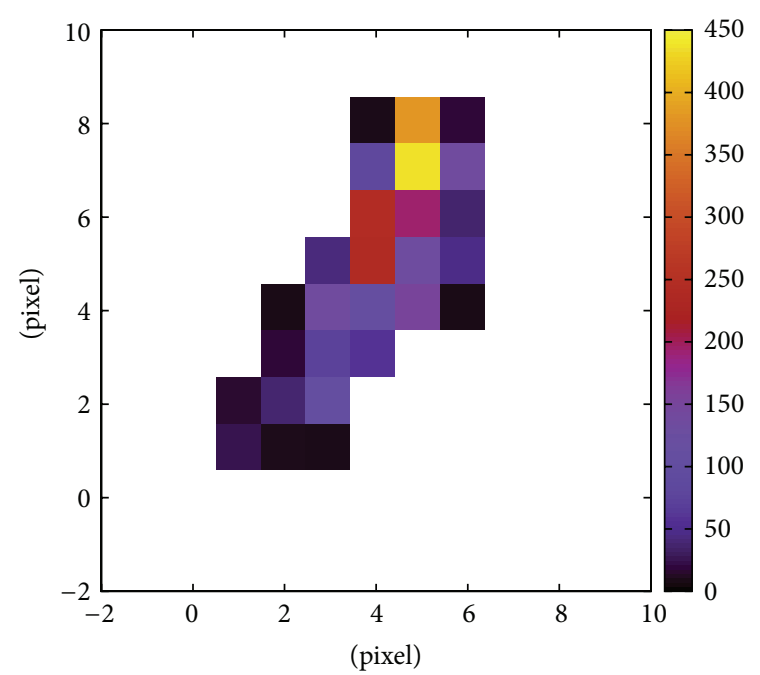

(b)

FIGURE 10: Simulated pattern of energy depositions in the pixel matrix for two examples of $0 \nu \beta \beta$-decay events. (a) Clear $0 \nu \beta \beta$-decay appearance. (b) event that looks like a single-electron track. The $x$ - and $y$-coordinates give the pixel row and column. The color bar encodes the energy deposition measured with the time-over-threshold method in keV.

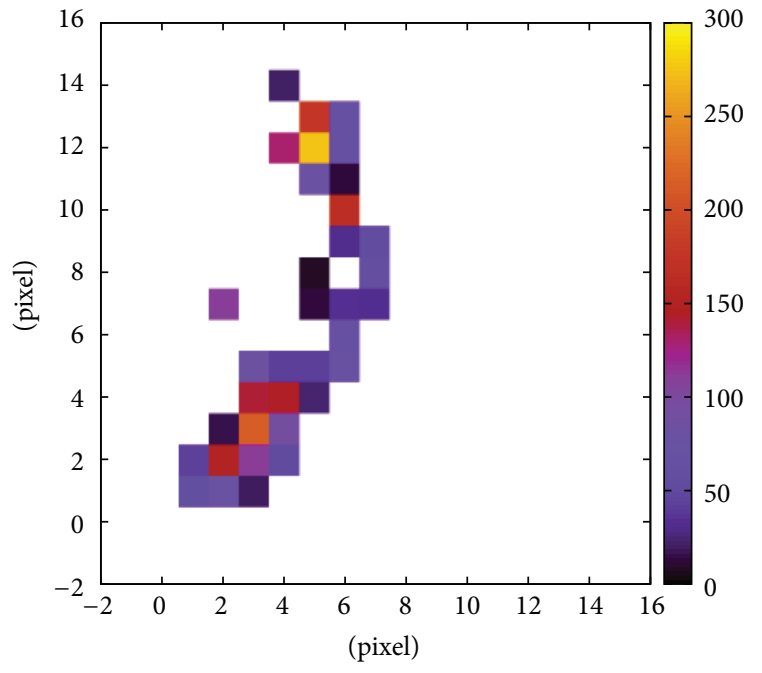

(a)

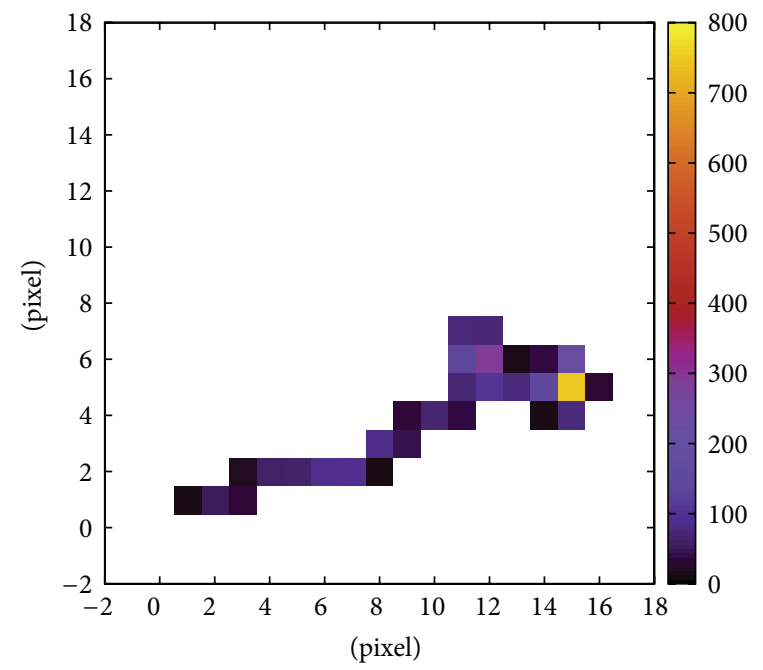

(b)

FIGURE 11: Simulated pattern of energy depositions in the pixel matrix of single electrons with energy deposition close to $E_{\mathrm{Q}}$. (a) Electron that has an appearance similar to a $0 \nu \beta \beta$-decay event. (b) Electron track like it is expected in a naive picture. The $x$-and $y$-coordinates give the pixel row and column. The color bar encodes the energy deposition measured with the time-over-threshold method in keV.

number of segments of the track that appeared as straight lines became a feature (Figure 13(b)). We defined a straight line as follows: there must be a pixel (starting point) that had collected less than $100 \mathrm{keV}$ energy and the remaining part of the track is found mainly in one direction if it is viewed from the starting point. A valid line was counted if two additional conditions were met. First, a triggered pixel (end point) had to be present that was connected with the starting point by at least a four-pixel long line of neighboring or diagonal pixels. Second, less than four pixels should be connected to the line (neighbors or diagonal) so that the line was not too broad. If a starting point had more than one valid end point, the end point with the largest distance to the starting point was chosen. If one line was identified in the track, the starting points and end points of further valid lines had to have a certain minimum distance to the lines identified before.

Tracks of single electrons often appear as thinner tracks than $0 \nu \beta \beta$-events. In one feature (Figure 13(c)) we counted the number of pixels which are part of a thin track segment, which was defined as a structure where the pixel had only two triggered neighbors on each side.

In an additional feature characterizing the track thickness (Figure 13(d)) we counted the number of pixels which had 

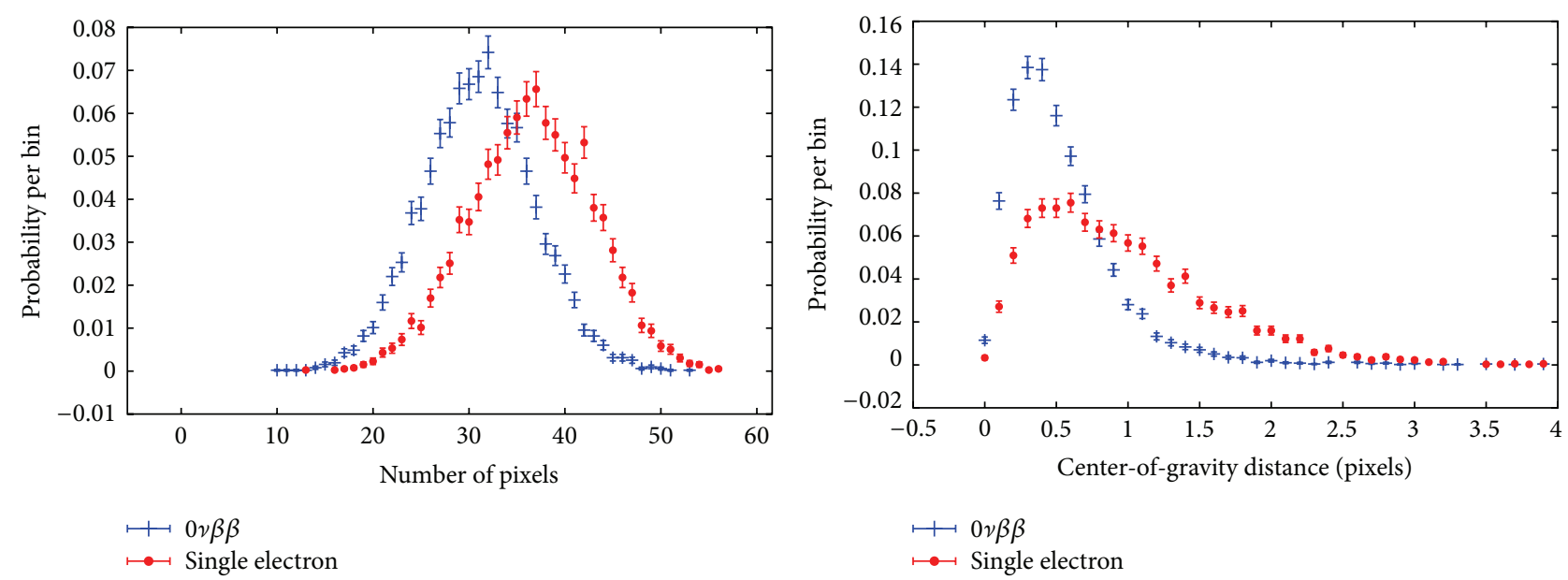

$\longmapsto 0 \nu \beta \beta$
$\longmapsto$ Single electron

(a) Number of pixels (bin width: 1 pixel)

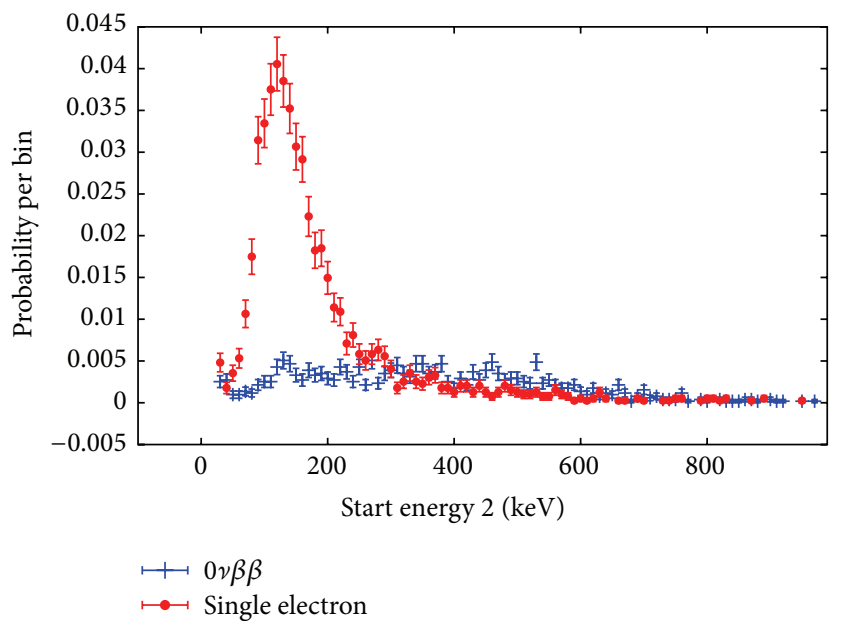

(b) Center-of-gravity distance (bin width: 0.1 pixel)

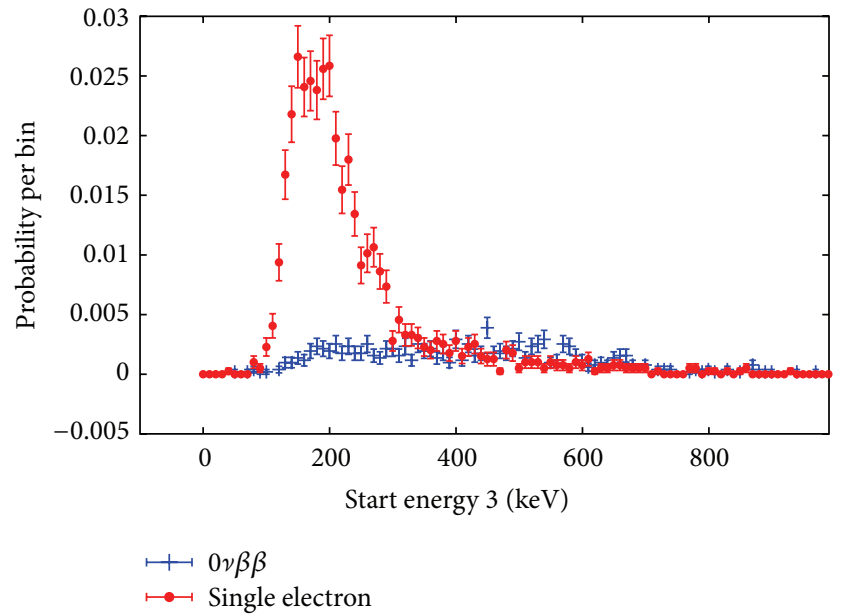

(c) Start energy 2 (bin width: $10 \mathrm{keV}$ )

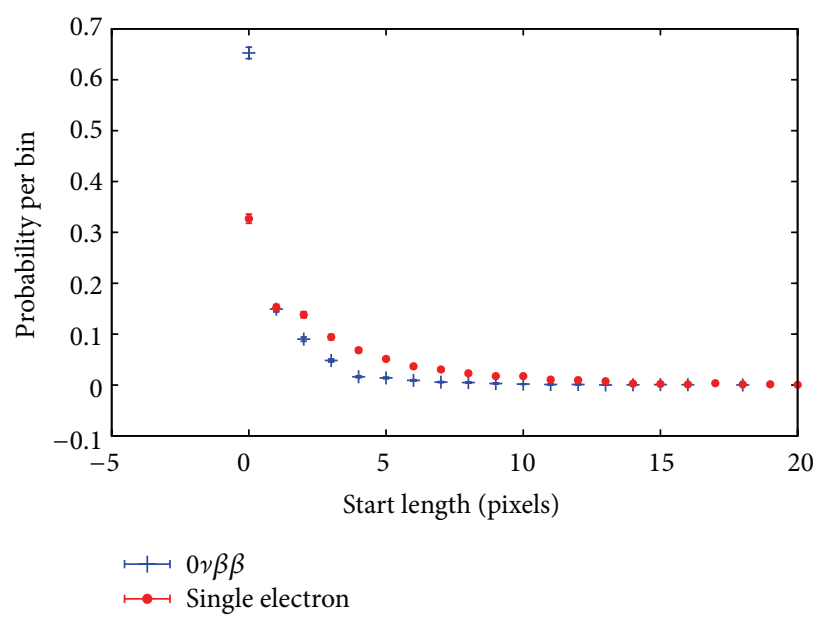

(e) Start length (bin width: 1 pixel)

(d) Start energy 3 (bin width: $10 \mathrm{keV}$ )

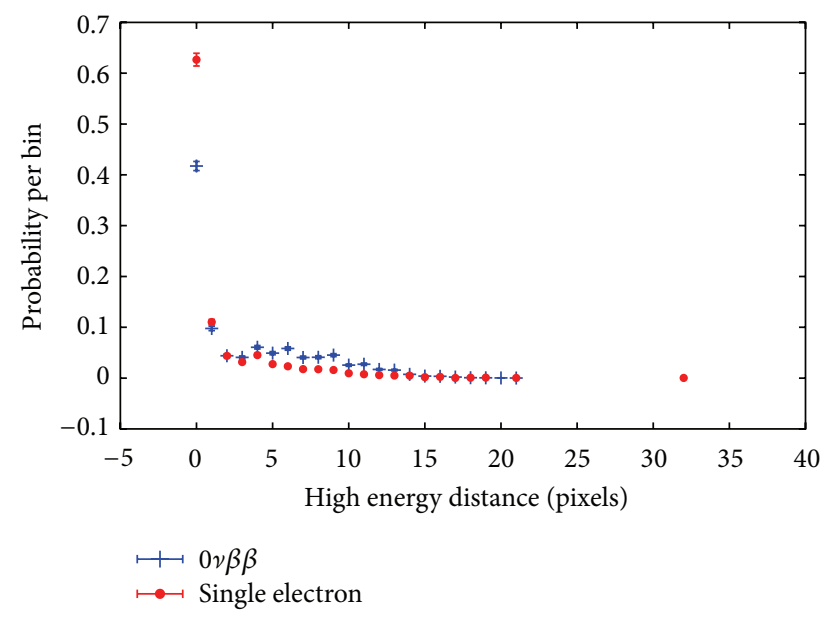

(f) High energy distance (bin width: 1 pixel)

FIGURE 12: Probabilities per bin for the occurrence of feature values in an event for $0 \nu \beta \beta$-decays and for single electrons with energy deposition around $E_{\mathrm{Q}}$ for a $3 \mathrm{~mm}$ thick CdTe sensor with $110 \mu \mathrm{m}$ pixel pitch. The bin width is given in the caption of each feature. 


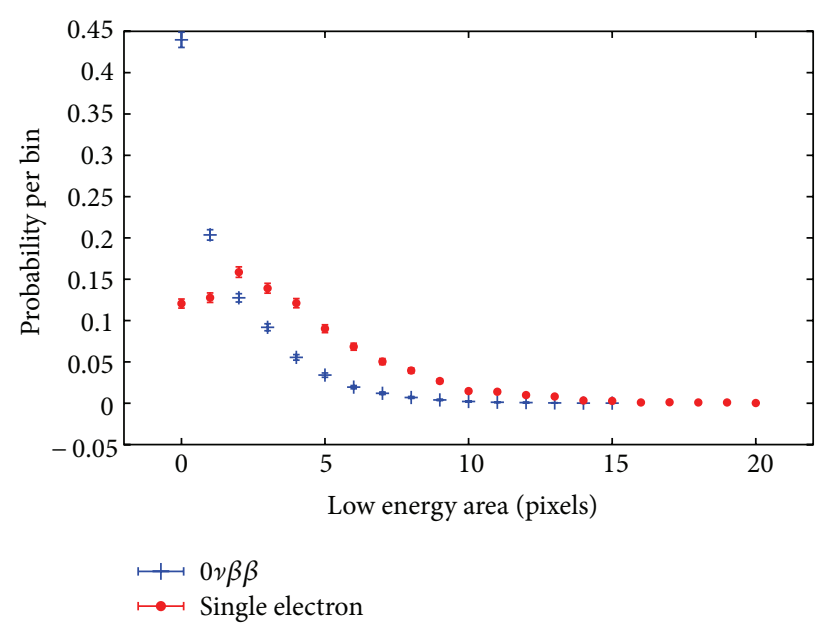

(a) Low energy area (bin width: 1 pixel)

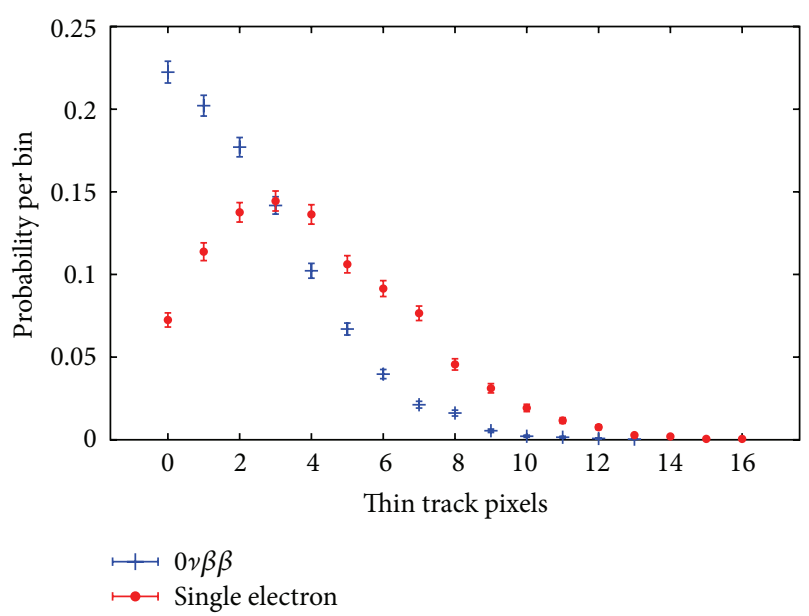

(c) Thin track pixels (bin width: 1 pixel)

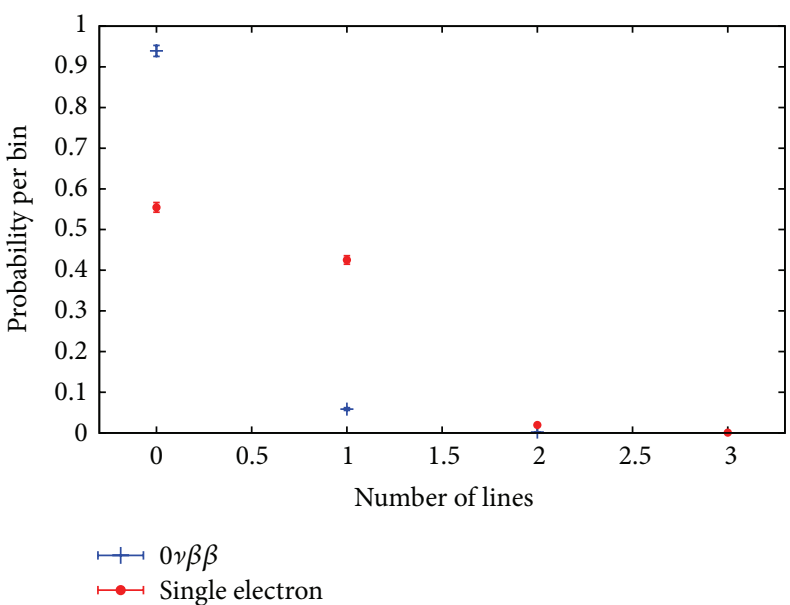

(b) Number of lines (bin width: 1 line)

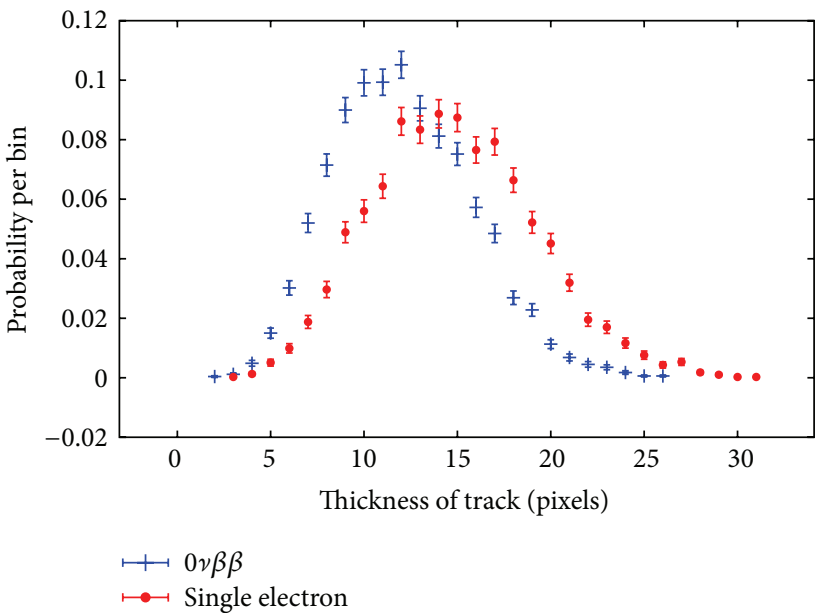

(d) Thickness of track (bin width: 1 pixel)

FIGURE 13: Probabilities per bin for the occurrence of feature values in an event for $0 \nu \beta \beta$-decays and for single electrons with energy deposition around $E_{\mathrm{Q}}$ for a $3 \mathrm{~mm}$ thick CdTe sensor with $110 \mu \mathrm{m}$ pixel pitch. The bin width is given in the caption of each feature.

two triggered neighbors so that the three pixels formed a triangle.

Many electrons that enter the sensor from the edges could be vetoed during data analysis with very high efficiency by using the columns and rows at the edges as vetoes. The collision stopping power of a $2.8 \mathrm{MeV}$ electron in CdTe is $1.161 \mathrm{MeV} \mathrm{cm}^{2} / \mathrm{g}$ [44] which translates to an average deposited energy of $113 \mathrm{keV}$ in a $165 \mu \mathrm{m}$-wide pixel. This energy deposition is well above the possible discriminator threshold. It would be favorable to use two adjacent pixel columns or rows as vetoes because pixels at the edges of the sensor sometimes suffer from increased leakage currents. Another possibility would be reading the charge signal from a field-forming guard ring at the edges of the sensor. Thus, fiducializing the sensor volume from the edges is possible and would reduce the single-electron background further.

3.7. Sensitivity to the Effective Majorana Neutrino Mass $m_{\beta \beta}$. The sensitivity of a large-scale experiment based on hybrid pixel detectors with a total CdTe mass of $420 \mathrm{~kg}$
(Cd enriched to $90 \%$ in ${ }^{116} \mathrm{Cd}$ ) was determined for different pixel pitch and sensor thickness combinations. Background and efficiency reduction with track analysis was accounted for. $2 \nu \beta \beta$-background and the single-electron background were taken into account. The spectrum of the single-electron background was modeled as a flat distribution in the region of $E_{\mathrm{Q}}$. The specific single-electron background rate was a free parameter. The underlying classification process with the random decision forests was optimized for a pixel pitch of $110 \mu \mathrm{m}$. The sensitivity of the setup was determined with the "simple and unambiguous statistical recipe" [41] of the unified approach, based on the Feldman-Cousins algorithm [45]. We derived half-life sensitivities for a confidence level of $90 \%$ reached after 5 years of measurement.

The cut width $\Delta E_{\text {cut }}$ on the deposited energy sum (for counting the number of signal events) in the track structure around $E_{Q}$ was optimized for maximum sensitivity for each combination of pixel pitch and sensor thickness. Figure 14 gives the results of this complex optimization. It can be seen that the best overall performance over a wide range of 


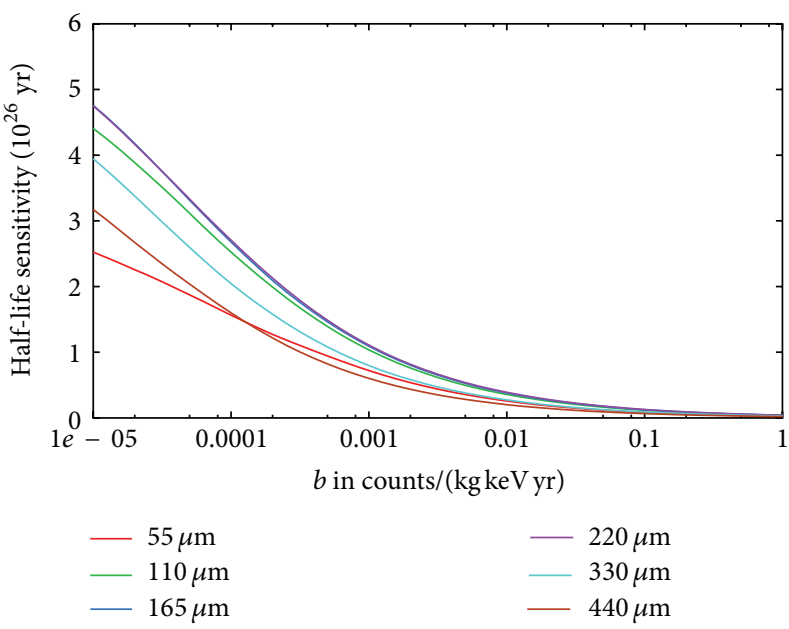

(a) $2 \mathrm{~mm}$ thickness

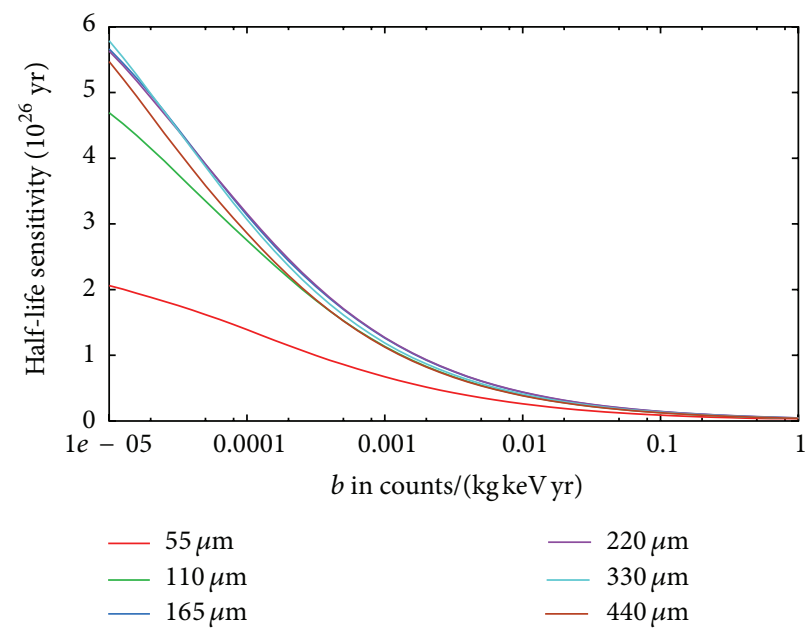

(c) $4 \mathrm{~mm}$ thickness

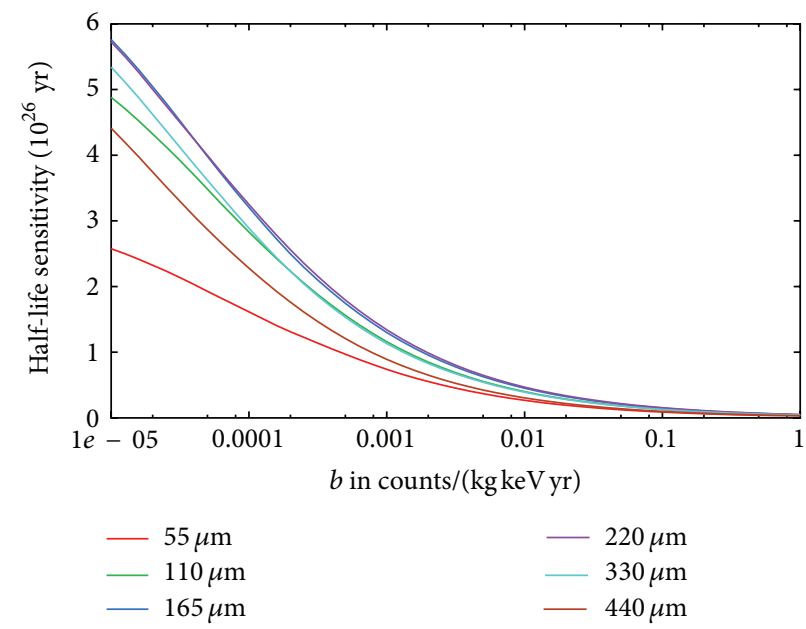

(b) $3 \mathrm{~mm}$ thickness

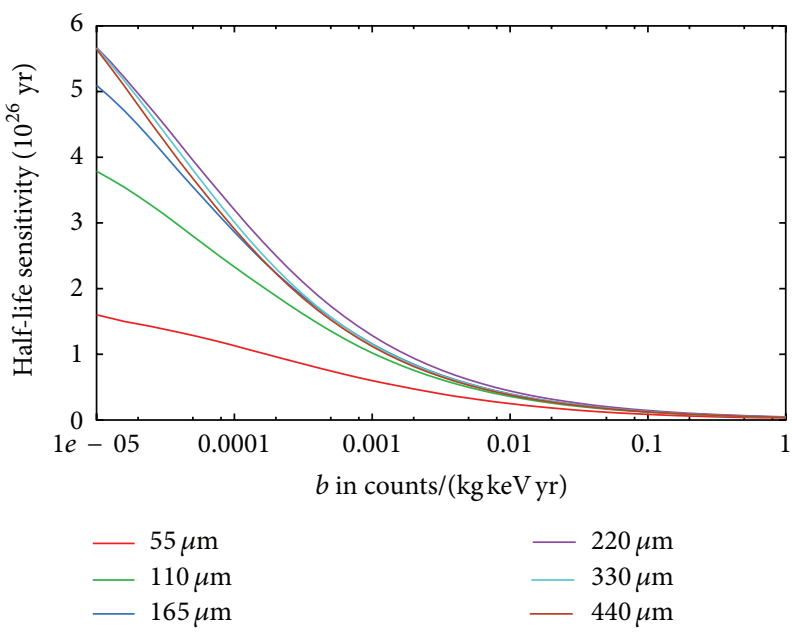

(d) $5 \mathrm{~mm}$ thickness

FIGURE 14: Simulated half-life sensitivity according to the unified approach [41] for $90 \%$ confidence level after 5 years of measuring time with $420 \mathrm{~kg}$ CdTe employing background identification for different combinations of sensor thickness and pixel size as functions of the specific single-electron background rate $b$.

background rates can be achieved with a $3 \mathrm{~mm}$ thick CdTe sensor layer and pixel pitch between $165 \mu \mathrm{m}$ and $220 \mu \mathrm{m}$. At a background level of $b=0.001$ counts $/(\mathrm{kg} \times \mathrm{keV} \times \mathrm{yr})$ the best half-life sensitivity of $1.34 \times 10^{26} \mathrm{yr}$ is obtained for a $3 \mathrm{~mm}$ thick sensor with $220 \mu \mathrm{m}$ pixel size. This sensitivity is close to the half-life sensitivity of $1.30 \times 10^{26} \mathrm{yr}$ obtained for the same sensor thickness but with $165 \mu \mathrm{m}$ pixel size. In our opinion, a pixel pitch of $165 \mu \mathrm{m}$ should be chosen in the experiment in order to obtain the better resolution of track structures.

Figure 15 shows the relative reduction $\Delta S(b) / S(b)$ of the signal and of the electron background $\Delta B / B(b)$ achieved with the track analysis. The signal $S$ was defined as the number of detected $0 \nu \beta \beta$-decay events in the signal acceptance window around $E_{\mathrm{Q}}$. The background $B$ was the corresponding number of detected electron background events in the acceptance window. $b$ is the specific background rate (given in counts $/(\mathrm{kg} \times \mathrm{keV} \times \mathrm{yr}))$ of single electrons before application of the random decision forests. The higher the background, the more restrictively the classification programs reacted.
Thus, the detection efficiency was reduced more at higher background rates. For $3 \mathrm{~mm}$ thick sensors with $110 \mu \mathrm{m}$ pixel pitch the detection efficiency amounted to $55 \%$ before event classification with the random decision forests. A reduction of the detection efficiency of about $23 \%$ occurred at a background level of about $10^{-3}$ counts $/(\mathrm{kg} \times \mathrm{keV} \times \mathrm{yr})$ so that a detection efficiency of $42 \%$ was reached after classification. At the same time, the electron background was reduced by $75 \%$.

The net benefit of track analysis could be represented by an effective background reduction factor $r$, which we defined as

$$
r=\frac{b_{\mathrm{RDF}}}{b_{\mathrm{noRDF}}} .
$$

$b_{\mathrm{RDF}}$ and $b_{\text {noRDF }}$ were the maximum allowed single-electron specific background rates $b$ to reach an effective Majorana neutrino mass sensitivity $\widetilde{m}_{\beta \beta}$ with (RDF) and without (noRDF) event classification in $0 \nu \beta \beta$-decays and single 


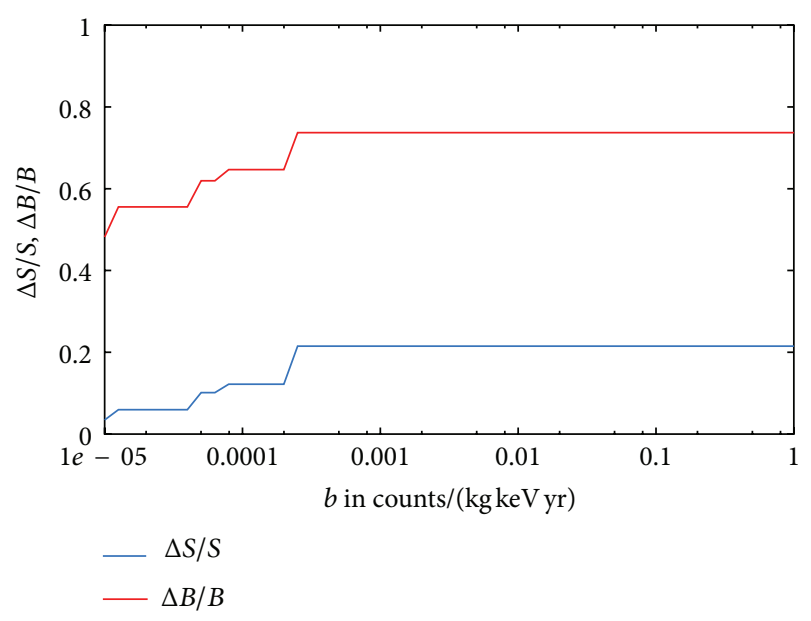

FigURE 15: Relative reduction of the number of detected signal events $\Delta S(b) / S(b)$ and the number of single-electron background events $\Delta B / B(b)$ in the acceptance window as functions of the original specific single-electron background rate (given in counts/ $(\mathrm{kg} \times$ $\mathrm{keV} \times \mathrm{yr}$ )) for a $3 \mathrm{~mm}$ thick CdTe sensor with $165 \mu \mathrm{m}$ pixel pitch achieved with the random decision forest.

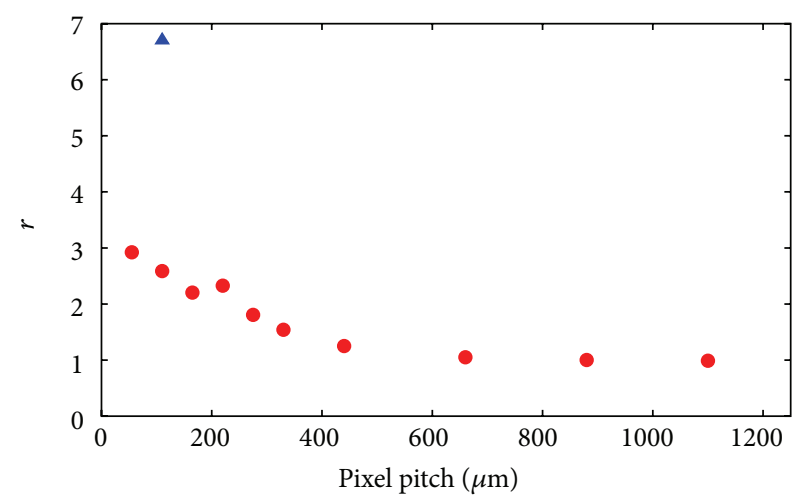

2D pixel detector

- 3D voxel detector

FIGURE 16: The effective background reduction factor $r$ as a function of pixel pitch after the classification working only in the sensor plane (red) for a $3 \mathrm{~mm}$ thick CdTe sensor. Additionally, the effective background reduction factor $r$ obtained with 3D-track analysis at $110 \mu \mathrm{m}$ pixel pitch is shown (blue).

electrons by the random decision forests. For $b_{\text {noRDF }}$ the cut on the $0 \nu \beta \beta$ total energy peak (window-width $\Delta E_{\text {cut }}$ and position) was optimized for maximum sensitivity to the effective Majorana neutrino mass. The random decision forest was not applied to discriminate between $0 \nu \beta \beta$ - and single-electron tracks. For $b_{\mathrm{RDF}}$, the random decision forest additionally classified events and rejected background events at the expense of signal efficiency. $r>1$ means that a larger single-electron background rate in the experiment can be tolerated with event classification by the random decision forest compared to the same experimental setup without using the random decision forest. Thus, $r$ reflects the potential of the event classification with the random decision forest with respect to the allowed background rate. $r$ measures by how much the background rate needs to be reduced without performing background rejection with the random decision forest so that the same sensitivity to the effective Majorana neutrino mass can be achieved as with background rejection with the random decision forest. The classification of events with the random decision forest during data analysis effectively reduces the single-electron background to $b_{\text {noRDF }} / r$.

We used the definition of the effective Majorana neutrino mass sensitivity $\widetilde{m}_{\beta \beta}$ given in (4.15) of [41]

$$
\widetilde{m}_{\beta \beta}=\frac{K_{2}}{\sqrt{\varepsilon}}\left(\frac{b \Delta E_{\text {cut }}}{M t}\right)^{1 / 4}
$$

which is an appropriate definition for large background levels. $K_{2}$ is a constant that depends only on the isotope, $\varepsilon$ is the corresponding detection efficiency, $M$ is the total mass of the double-beta decay nuclei in the detector, $b$ is the singleelectron specific background rate in counts $/(\mathrm{kg} \times \mathrm{keV} \times \mathrm{yr})$, and $t$ is the measuring time. $\Delta E_{\text {cut }}$ specifies the acceptance window width around the $0 v \beta \beta$-decay peak in the spectrum of total energies.

Figure 16 shows the effective background reduction factor $r$ as a function of pixel pitch for a $3 \mathrm{~mm}$ thick CdTe sensor layer. It can be seen that the track analysis has a greater benefit in an experiment with smaller pixel pitch and that almost no benefit with track analysis can be achieved for pixel sizes larger than $440 \mu \mathrm{m}$. One can see that the exploitation of the tracking properties of a detector with a pixel pitch of $165 \mu \mathrm{m}$ reduces the single-electron background level by a factor of 2.2. The sensitivity to the effective Majorana neutrino mass is thus improved by a factor of 1.22 for the optimal sensor layer configuration ( $3 \mathrm{~mm}$ thickness, $165 \mu \mathrm{m}$ pitch).

Figure 17 shows the expected sensitivity in terms of effective Majorana neutrino mass of a $420 \mathrm{~kg}$ CdTe experiment after 5 years of measuring time for the optimal sensor configuration with track analysis, as a function of the singleelectron background rate $b$ before event classification, in the region of $E_{\mathrm{Q}} .2 \nu \beta \beta$-background was taken into account. Our simulations showed that a single-electron background level of about $2 \times 10^{-4}$ in the region of $E_{\mathrm{Q}}$ would be necessary to access the effective Majorana neutrino masses in the inverted hierarchy range-which begins at $m_{\beta \beta} \approx 50 \mathrm{meV}$-in 5 years of measuring time at $90 \%$ confidence level.

3.8. Discrimination of $\alpha$-Particle Background. Figure 18 shows a measured "track" of an $\alpha$-particle with $5.5 \mathrm{MeV}$ ki-netic energy impinging on the common electrode side. With its approximate radial symmetry and high energy deposition in the center it looks completely different to the majority of the $0 \nu \beta \beta$-tracks. The track lengths of $\alpha$-particles with energies close to the $Q$-value of the $0 \nu \beta \beta$-decay are very short in CdTe (few micrometers) compared to the sum of the track lengths of the two $0 \nu \beta \beta$-electrons (several hundred micrometers) and compared to the pixel pitches under investigation. Thus, we can assume in the simulation of $\alpha$-particle signatures that the kinetic energy is deposited at one point. The dynamics of the very dense charge carrier distribution along the short track of an $\alpha$-particle 


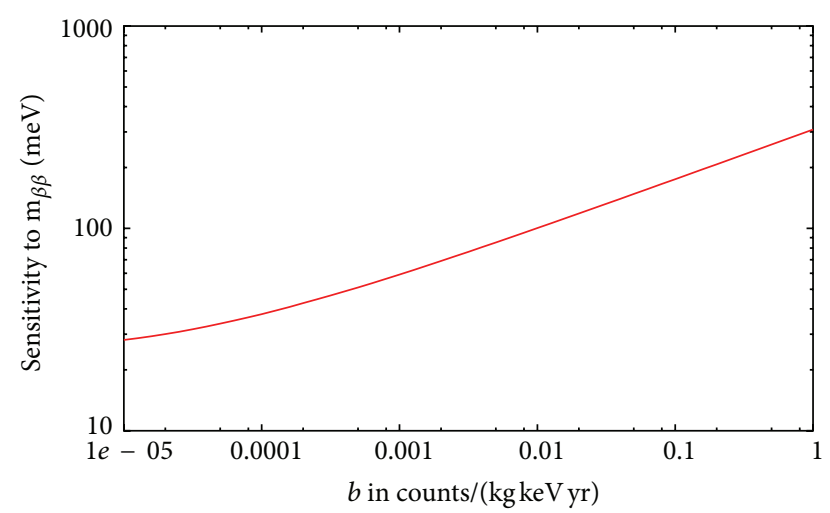

FIGURE 17: Expected sensitivity to the effective Majorana neutrino mass $m_{\beta \beta}$ for the optimal configuration ( $3 \mathrm{~mm}$ thickness and $165 \mu \mathrm{m}$ pixel pitch) as a function of the specific single-electron background rate $b$ before track analysis given in counts $(\mathrm{kg} \times \mathrm{keV} \times \mathrm{yr})$.

immediately after impact of the particle is complicated because of space charge buildup which partly compensates the electric drift field. This leads to a period of diffusion without significant drift during the early history of the detection process. Additionally, charge carrier repulsion takes place. The result is that the pattern of triggered pixels becomes larger than one would expect. An experiment with a $1 \mathrm{~mm}$ thick CdTe sensor with $110 \mu \mathrm{m}$ pixel pitch revealed that 20-60 pixels are triggered by impacts of $5.5 \mathrm{MeV} \alpha$ particles, depending on the bias voltage, which was varied between $-100 \mathrm{~V}$ and $-500 \mathrm{~V}$. We introduced a factor into the simulation which broadened the distribution of drifted charge carriers. We chose a large range for this value in the simulation, so that the measured distributions of cluster sizes for the $5.5 \mathrm{MeV} \alpha$-particles were covered widely with the simulations for the different bias voltages. We then simulated signatures of $2.8 \mathrm{MeV}$ energy depositions-which were supposed to be caused by $\alpha$-particle impact on the common electrode side-for the whole range of this factor to cover all possible pattern sizes. We simulated $10^{6} \alpha$-particle impacts with a value for this factor which leads to $\alpha$-particle signatures that were most difficult to discriminate with the classification software. For this worst case the random decision forest, trained with simulated pattern of $\alpha$-particles, was able to reject all simulated $\alpha$-particle impacts with less than $2.6 \%$ loss of signal events $(0 \nu \beta \beta)$. We expect that the $\alpha$-particle background can be reduced further, because the classification algorithm did not have features dedicated to $\alpha$-particle identification (like symmetry of the topological pattern with the largest energy deposition in the center).

\section{Future Perspectives}

4.1. Tracking in Three Dimensions. The analysis presented so far only used two-dimensional projections of the energy deposition in the electron tracks for background discrimination, although an electron travels through the sensor in three dimensions. A long track segment of an electron through a pixel parallel to the axis of the electric field lines ( $z$-axis)

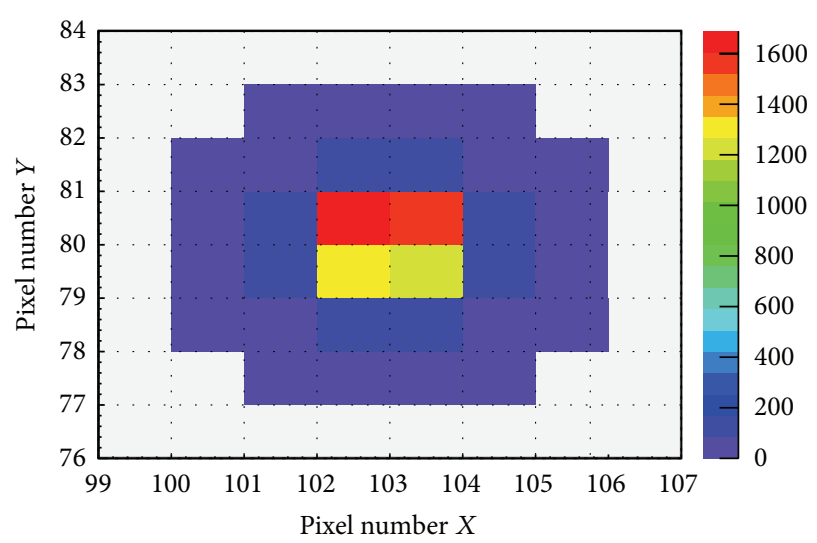

FIGURE 18: Measured image of the energy deposition of a $5.8 \mathrm{MeV}$ $\alpha$-particle impact on the common electrode side, recorded with a $110 \mu \mathrm{m}$ pixel pitch Timepix detector with a $1 \mathrm{~mm}$ thick sensor layer.

causes large energy deposition similar to a less energetic electron travelling perpendicular to the electric field lines. As a consequence of this, some single-electron tracks are difficult to distinguish from signal events. This problem could be overcome if a 3D tracking hybrid pixel detector would be available. Information about the $\mathrm{z}$-components of a track could in principle be obtained by measuring differences in drift times of charge carrier distributions collected by neighboring pixels. Campbell et al. [46] have proposed a schematic of such an architecture of a pixel detector which avoids the distribution of a fast clock signal for timing measurements to all pixels. A fast clock signal supplied to all pixels typically generates noise that in turn deteriorates energy resolution and increases the minimum discriminator threshold. Another possibility for an architecture would be the combination of a coarse time stamp generated by a slow clock signal distributed to all pixels in the matrix and a precise time stamp generated by a fast oscillator in each pixel. This architecture is implemented in the Timepix3 detector [16].

We have carried out simulations to demonstrate the potential of $3 \mathrm{D}$ tracking with the assumption that a position resolution of $110 \mu \mathrm{m}$ could be achieved in each pixel cell also in the drift direction. This means that $2.7 \mathrm{~ns}$ resolution of drift time measurements is needed. This does not seem to be impossible because the clock cycle spacing of the fast oscillator signal in the Timepix 3 is 1.6 ns. The preamplifier peaking time in the Timepix 3 is less than $20 \mathrm{~ns}$ and the time over threshold is provided so that time walk corrections can be carried out. The electron drift time to cross the complete sensor thickness of $3 \mathrm{~mm}$ is $73.5 \mathrm{~ns}$ for a bias voltage difference of $-1500 \mathrm{~V}$. As a simple test of $3 \mathrm{D}$ track analysis, we applied the two-dimensional track analysis presented above three times for each plane in the sensor $(x-y, x-z, y$ $z$ ) independently from each other. The result, expressed by the equivalent background reduction factor $r$, is shown in Figure 15 (blue). One can see that with a 3D voxel detector an increase of the sensitivity to the effective Majorana neutrino mass by a factor of about 1.61 with track analysis could be 
realized. This would be a significant improvement compared to the $2 \mathrm{D}$ pixel detector. We expect an even stronger increase of the sensitivity to the effective Majorana neutrino mass by analysis of additional topological features which take the three-dimensional structure into account.

\subsection{Improvement of Energy Resolution. Energy resolution} should be further improved. In contrast to photon counting X-ray imaging or accelerator-based high-energy-physics experiments, an analog read-out of the energy signal of pixels with external ADCs is possible in low-background experiments because of the low overall detection rate. It has been demonstrated [47] that an excellent average single-pixel energy resolution of $\Delta E_{\mathrm{FWHM}} / E=1.2 \%$ at $59.5 \mathrm{keV}$ can be achieved with the hybrid pixel detector HEXITEC with $250 \mu \mathrm{m}$ pixel pitch bump-bonded to a $1 \mathrm{~mm}$ thick CdTe layer with Schottky contacts biased to $-500 \mathrm{~V}$. Seller et al. [21] reported an energy resolution better than $2.5 \%$ (FWHM) at $59.5 \mathrm{keV}$ on a $2 \mathrm{~mm}$ thick $20 \times 20$ pixel CdZnTe detector with $250 \mu \mathrm{m}$ pixel pitch connected to an ASIC with a pixel circuitry identical to the HEXITEC. In the HEXITEC detector, the energy signal is digitized externally with a low-noise ADC. The drawback of this pixel detector is that no differences of drift times can be obtained. The HEXITEC detector lacks time resolution on a few-nanosecond scale, which is provided by the Timepix detector. Such a time resolution seems to be necessary in order to be sure that potential double-beta decay events that come from one single decay are not due to a random coincidence of two single beta decays. Time resolution will already be improved with the Timepix 3 active pixel detector. Each $55 \times 55 \mu \mathrm{m}^{2}$ pixel of the Timepix 3 detector [16] features an amplifier with less than $20 \mathrm{~ns}$ peaking time. A fast oscillator in each pixel provides a clock pulse each $1.6 \mathrm{~ns}$ for in-pixel time stamping if the signal pulse height is above discriminator threshold. In our opinion an analog-electronics pixel architecture feeding an analog read-out chain with external low-noise ADCs (like in the HEXITEC detector) should be combined with a fast timing circuit in each pixel (like in the Timepix3). This combination of a fast and a slow circuit might require the generation of two signal copies on the pixel level. The Medipix3 [48] detector already features such a circuit in each pixel which generates four copies of the amplified sensor signal for reallocation of the charge which is split between neighboring pixels.

\section{Conclusion}

We have evaluated the sensitivity of an experiment based on pixel detectors connected to pixelated sensors with $420 \mathrm{~kg}$ of CdTe (Cd enriched to $90 \%$ in ${ }^{116} \mathrm{Cd}$ ), arranged in face-toface geometry of assemblies $\left(8 \mathrm{~cm}^{2}\right.$ area) for the measurement of the effective Majorana neutrino mass. For the time-and energy-resolving hybrid pixel detector Timepix we found an optimal thickness of the sensor layer of $3 \mathrm{~mm}$ and a pixel pitch of $165 \mu \mathrm{m}$. With the mass density of enriched CdTe of approximately $5.9 \mathrm{~g} / \mathrm{cm}^{3}$, this translates to a sensor area of $2 \times 11.9 \mathrm{~m}^{2}$ with about 875 million pixels. We found that $\alpha$ particle background was reduced by at least about 6 orders of magnitude due to the different track structures of $\alpha$-particles and electrons.

We found that the single-electron background rate could be reduced with track analysis by about $75 \%$ at a single-electron background level of $10^{-3}$ counts $/(\mathrm{kg} \times \mathrm{keV} \times$ yr). Track analysis with random decision forests reduced the effective single-electron background by a factor of 2.2. This corresponds to an improvement of the Majorana mass sensitivity by a factor of 1.22. A Majorana mass sensitivity of $59 \mathrm{meV}$ on a $90 \%$ confidence level according to the unified approach [41] might be reached after 5 years of measuring time for a specific single-electron background rate of $10^{-3}$ counts/ $(\mathrm{kg} \times \mathrm{keV} \times \mathrm{yr})$. In our opinion, further improvements in sensitivity can be achieved with external digitization of the energy signal instead of the current in-pixel conversion based on the time-over-threshold method.

Currently, gaseous detectors outperform the background identification potential of the semiconductor pixel detector concept for $0 \nu \beta \beta$-decay detection because of the much longer track length and their possibility of $3 \mathrm{D}$ track reconstruction. It was shown within the framework of the NEXT experiment [49] that background can be reduced by 3 orders of magnitude with tolerable reduction of the signal detection efficiency by analysis of tracks in a high-pressure Xenon TPC. Further improvement of the sensitivity to $m_{\beta \beta}$ with $\mathrm{CdTe}$ pixel detectors will be achieved with track analysis exploiting features of the three-dimensional electron tracks if precise timing measurements of drift time differences between adjacent pixels allow $3 \mathrm{D}$ reconstruction of electron tracks.

\section{Acknowledgments}

The authors cordially thank the COBRA collaboration for intense research on $\mathrm{CdTe}$ and $\mathrm{CZT}$ detectors and for supporting this study of the pixel detector option. They thank the Medipix collaboration for the support and for the development of the Timepix detector. They thank the Deutsche Forschungsgemeinschaft DFG for supporting Thomas Gleixner in the project MI 1507/1-1.

\section{References}

[1] J. Schechter and J. W. F. Valle, "Neutrinoless double-decay in $\mathrm{SU}(2) \times \mathrm{U}(1)$ theories," Physical Review D, vol. 25, no. 11, pp. 2951-2954, 1982.

[2] H. Nunokawa, W. J. C. Teves, and R. Z. Funchal, "Constraining the absolute neutrino mass scale and Majorana $\mathrm{CP}$ violating phases by future neutrinoless double beta decay experiments," Physical Review D, vol. 66, no. 9, Article ID 093010, 2002.

[3] R. Arnold, C. Augier, J. Baker et al., "Probing new physics models of neutrinoless double beta decay with SuperNEMO," European Physical Journal C, vol. 70, no. 4, pp. 927-943, 2010.

[4] J. J. Gómez-Cadenas, J. Martín-Albo, M. Mezzetto, F. Monrabal, and M. Sorel, "The search for neutrinoless double beta decay," Rivista del Nuovo Cimento, vol. 35, no. 2, pp. 29-98, 2012.

[5] The NEXT Collaboration, "Initial results of NEXT-DEMO, a large-scale prototype for the NEXT-100 experiment," Journal of Instrumentation, vol. 8, Article ID P04002, 2013. 
[6] S. Schinert, I. Abt, M. Altmann et al., "The GERmanium Detector Array (GERDA) for the search of neutrinoless $2 \beta$ decays of ${ }^{76}$ Ge at LNGS," Nuclear Physics B, vol. 145, pp. 242-245, 2005.

[7] D. G. Phillips II, D. G. Phillips II, E. Aguayo et al., "The Majorana experiment: an ultra-low background search for neutrinoless double-beta decay," Journal of Physics: Conference Series, vol. 381, conference 1, Article ID 012044.

[8] K. Zuber, "COBRA-double beta decay searches using CdTe detectors," Physics Letters B, vol. 519, no. 1-2, pp. 1-7, 2001.

[9] J. Ebert, M. Fritts, C. Gößling et al., "Current status and perspectives of the COBRA experiment," Advances in High Energy Physics, vol. 2013, Article ID 703572, 6 pages, 2013.

[10] P. Cermak, I. Stekl, V. Bocarov et al., "Background capabilities of pixel detectors for double beta decay measurements," Nuclear Instruments and Methods in Physics Research A, vol. 633, no. 1, pp. S210-S211, 2011.

[11] P. Cermak, I. Stekl, Y. A. Shitov et al., "Use of silicon pixel detectors in double electron capture experiments," Journal of Instrumentation, vol. 6, no. 1, Article ID C01057, 2011.

[12] J. Suhonen and O. Civitarese, "Weak-interaction and nuclearstructure aspects of nuclear double beta decay," Physics Report, vol. 300, no. 3-4, pp. 123-214, 1998.

[13] K. Zuber, "Neutrinoless double beta decay," Pramana Journal of Physics, vol. 79, pp. 781-791, 2012.

[14] Mathieu Bongrand for the NEMO-3 Collaboration, "Results of the NEMO-3 double betadecay experiment," http://arxiv .org/abs/1105.2435.

[15] F. A. Danevich, A. S. Georgadze, V. V. Kobychev et al., "Search for $2 \beta$ decay of cadmium and tungsten isotopes: final results of the Solotvina experiment," Physical Review C, vol. 68, no. 3, Article ID 035501, 12 pages, 2003.

[16] V. Gromov, M. van Beuzekom, R. Kluit et al., "Development and applications of the Timepix 3 readout chip," in Proceedings of 20th Anniversary International Workshop on Vertex detectors, PoS (Vertex 2011) 046, Rust, Austria, June 2011.

[17] X. Llopart, R. Ballabriga, M. Campbell, L. Tlustos, and W. Wong, "Timepix, a 65k programmable pixel readout chip for arrival time, energy and/or photon counting measurements," Nuclear Instruments and Methods in Physics Research A, vol. 581, no. 1-2, pp. 485-494, 2007.

[18] M. Campbell, "10 years of the Medipix2 collaboration," Nuclear Instruments and Methods in Physics Research A, vol. 633, no. 1, pp. S1-S10, 2011.

[19] D. Greiffenberg, A. Fauler, A. Zwerger, and M. Fiederle, "Energy resolution and transport properties of CdTe-Timepix-assemblies," Journal of Instrumentation, vol. 6, no. 1, Article ID C01058, 2011.

[20] M. Filipenko, T. Gleixner, G. Anton, J. Durst, and T. Michel, "Characterization of the energy resolution and the tracking capabilities of a hybrid pixel detector with CdTe-sensor layer for a possible use in a neutrinoless double beta decay experiment," The European Physical Journal C, vol. 73, article 2374, 2013.

[21] P. Seller, S. Bell, R. J. Cernik et al., "Pixellated Cd(Zn)Te highenergy X-ray instrument," Journal of Instrumentation, vol. 6, no. 12, Article ID C12009, 2011.

[22] H. Spieler, Semiconductor Detector Systems, Oxford Science Publications, 2005.

[23] X. L. Cudie, Design and characterization of $64 K$ pixels chips working in single photon processing mode [Ph.D. thesis], Department of Information Technology and Media Mid Sweden University.
[24] O. A. Ponkratenko, V. I. Tretyak, and Y. G. Zdesenko, "Event generator DECAY4 for simulating double-beta processes and decays of radioactive nuclei," Physics of Atomic Nuclei, vol. 63, no. 7, pp. 1282-1287, 2000.

[25] J. Giersch, A. Weidemann, and G. Anton, "ROSI-an objectoriented and parallel-computing Monte Carlo simulation for X-ray imaging," Nuclear Instruments and Methods in Physics Research A, vol. 509, no. 1-3, pp. 151-156, 2003.

[26] A. Castoldi, E. Gatti, and P. Rehak, "Three-dimensional analytical solution of the laplace equation suitable for semiconductor detector design," IEEE Transactions on Nuclear Science, vol. 43, no. 1, pp. 256-265, 1996.

[27] E. Guni, J. Durst, B. Kreisler et al., "The influence of pixel pitch and electrode pad size on the spectroscopic performance of a photon counting pixel detector with CdTe sensor," IEEE Transactions on Nuclear Science, vol. 58, no. 1, pp. 17-25, 2011.

[28] E. Guni, J. Durst, T. Michel, and G. Anton, "Material reconstruction with the Medipix2 detector with CdTe sensor," Journal of Instrumentation, vol. 6, no. 1, Article ID C01037, 2011.

[29] H. Spieler and E. Haller, "Assessment of present and future large-scale semiconductor detector systems," IEEE Transactions on Nuclear Science, vol. 32, no. 1, pp. 419-426, 1985.

[30] W. Shockley, "Currents to conductors induced by a moving point charge," Journal of Applied Physics, vol. 9, no. 10, pp. 635636, 1938.

[31] S. Ramo, "Currents induced by electron motion," Proceedings of the IRE, vol. 27, pp. 584-585, 1939.

[32] G. Knoll, Radiation Detection and Measurement, John Wiley \& Sons, New York, NY, USA, 4th edition, 2010.

[33] J. Jakubek, "Precise energy calibration of pixel detector working in time-over-threshold mode," Nuclear Instruments and Methods in Physics Research A, vol. 633, no. 1, pp. S262-S266, 2011.

[34] M. Platkevic, V. Bocarov, J. Jakubek, S. Pospisil, V. Tichy, and Z. Vykydal, "Signal processor controlled USB2.0 interface for Medipix2 detector," Nuclear Instruments and Methods in Physics Research A, vol. 591, no. 1, pp. 245-247, 2008.

[35] T. Holy, J. Jakubek, S. Pospisil, J. Uher, D. Vavrik, and Z. Vykydal, "Data acquisition and processing software package for Medipix2," Nuclear Instruments and Methods in Physics Research A, vol. 563, no. 1, pp. 254-258, 2006.

[36] S. Nissen, Implementation of a Fast Artificial Neural Network Library (FANN), Department of Computer Science, University of Copenhagen (DIKU), 2003.

[37] N. Remoué, D. Barret, O. Godet, and P. Mandrou, "Extensive testing of Schottky CdTe detectors for the ECLAIRs X-/gammaray camera on board the SVOM mission," Nuclear Instruments and Methods in Physics Research A, vol. 618, no. 1-3, pp. 199-208, 2010.

[38] S. del Sordo, L. Abbene, E. Caroli, A. M. Mancini, A. Zappettini, and P. Ubertini, "Progress in the development of CdTe and CdZnTe semiconductor radiation detectors for astrophysical and medical applications," Sensors, vol. 9, no. 5, pp. 3491-3526, 2009.

[39] T. Seino and I. Takahashi, "CdTe detector characteristics at 30॰C and $35 \circ \mathrm{C}$ when using the periodic bias reset technique," IEEE Transactions on Nuclear Science, vol. 54, no. 4, pp. 777-781, 2007.

[40] J. Fink, H. Krüger, P. Lodomez, and N. Wermes, "Characterization of charge collection in CdTe and CZT using the transient current technique," Nuclear Instruments and Methods in Physics Research A, vol. 560, no. 2, pp. 435-443, 2006. 
[41] J. J. Gómez-Cadenas, J. Martín-Albo, M. Sorel et al., "Sense and sensitivity of double beta decay experiments," Journal of Cosmology and Astroparticle Physics, vol. 2011, no. 6, article 007, 2011.

[42] T. K. Ho, "Random decision forests," in Proceedings of the $3 r d$ International Conference on Document Analysis and Recognition, pp. 278-282, 1995.

[43] ALGLIB Project (Free edition), Nizhny Novgorod, Russian Federation, http://www.alglib.net

[44] National Institute of Standards and Technology, "Stopping power and range tables for electrons," http://physics.nist.gov/ PhysRefData/Star/Text/ESTAR.html.

[45] G. J. Feldman and R. D. Cousins, "Unified approach to the classical statistical analysis of small signals," Physical Review D, vol. 57, no. 7, pp. 3873-3889, 1998.

[46] M. Campbell, J. Jakubek, and T. Michel, "A single layer 3D tracking semiconductor detector," Patent application WO 2013041114 A1, 2011.

[47] M. C. Veale, S. J. Bell, P. Seller, M. D. Wilson, and V. Kachkanov, "X-ray micro-beam characterization of a small pixel spectroscopic CdTe detector," Journal of Instrumentation, vol. 7, Article ID P07017, 2012.

[48] R. Ballabriga, M. Campbell, E. Heijne, X. Llopart, L. Tlustos, and W. Wong, "Medipix3: a 64 k pixel detector readout chip working in single photon counting mode with improved spectrometric performance," Nuclear Instruments and Methods in Physics Research A, vol. 633, no. 1, pp. S15-S18, 2011.

[49] S. Cebrian, T. Dafni, H. Gomez et al., "Pattern recognition of ${ }^{136} \mathrm{Xe}$ double beta decay events and background discrimination in a high pressure Xenon TPC," http://arxiv.org/abs/1306.3067. 

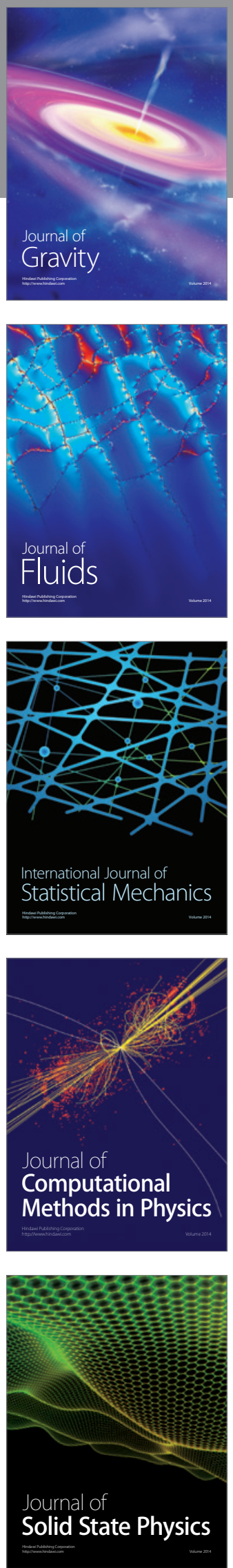

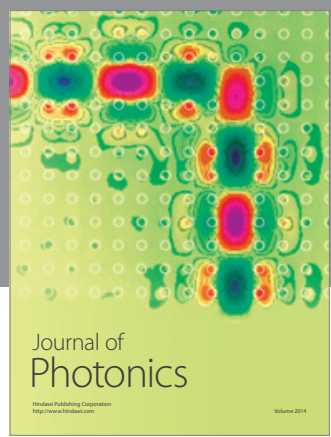

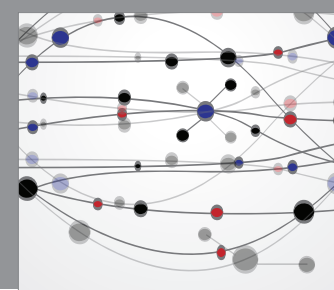

The Scientific World Journal

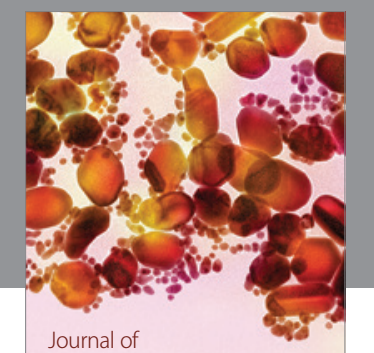

Soft Matter
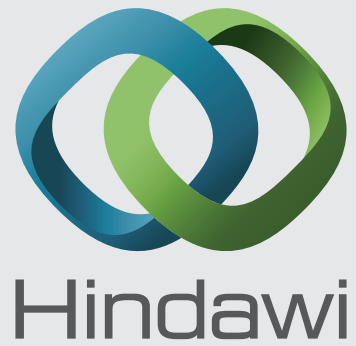

Submit your manuscripts at

http://www.hindawi.com
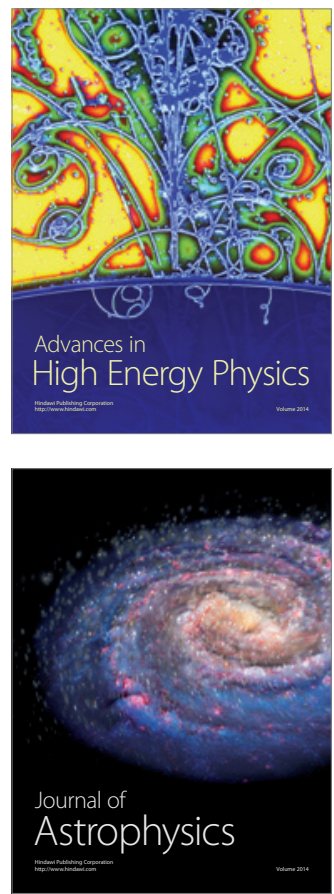
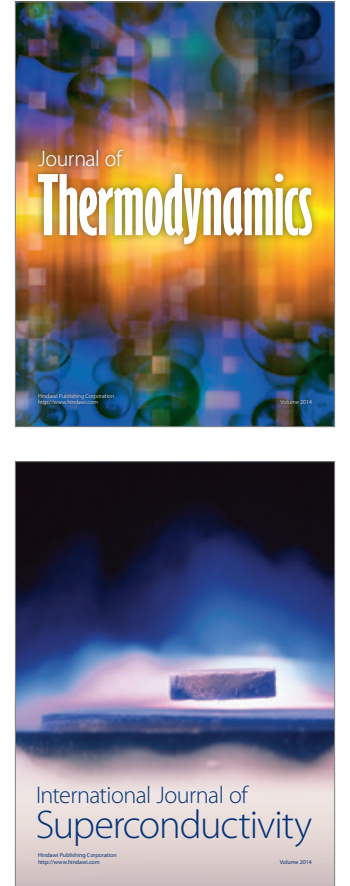
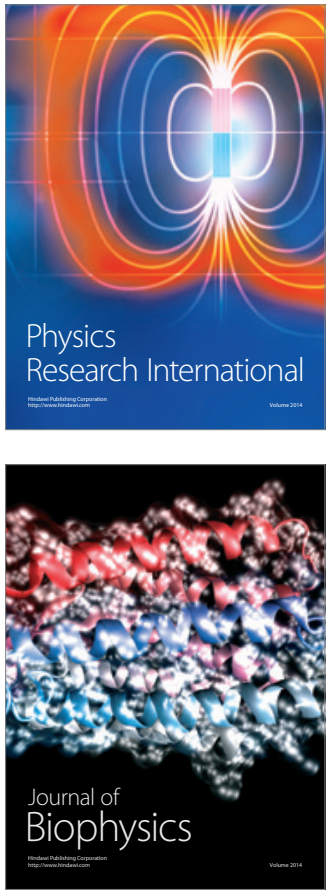
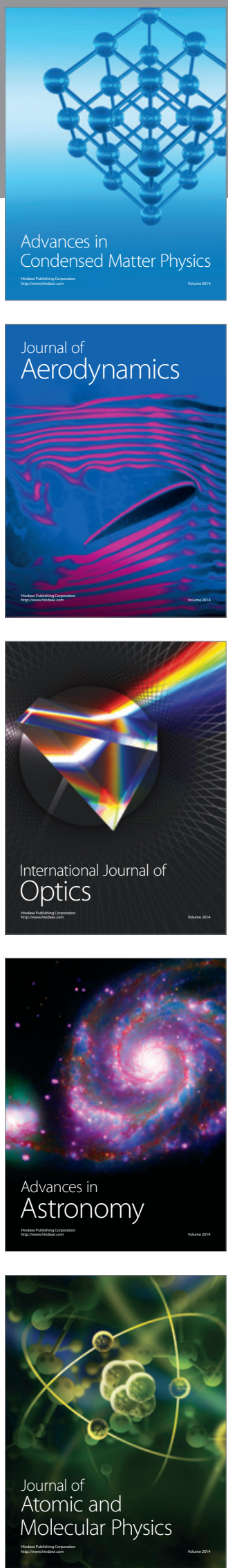